\title{
əThe Poisson Link between Internal Wave and Dissipation Scales in the Thermocline. Part I: Probability Density Functions and the Poisson Modeling of Vertical Strain
}

\author{
ROBERT PINKEL ${ }^{\text {a }}$ \\ ${ }^{a}$ Marine Physical Laboratory, Scripps Institution of Oceanography, University of California, San Diego, \\ La Jolla, California
}

(Manuscript received 26 November 2019, in final form 30 August 2020)

\begin{abstract}
The irregular nature of vertical density profiles is a ubiquitous characteristic of the ocean thermocline. This distortion can be quantified by tracking a set of constant-density (isopycnal) surfaces over time. Examination of $30000 \mathrm{~km}$ of vertical density profile data from seven Pacific Ocean sites indicates that the statistics of isopycnal vertical separation follow the gamma probability distribution, the continuous representation of a Poisson process. All aspects of this process are specified by a single parameter $\kappa_{0}$, of order $0.5-2 \mathrm{~m}^{-1}$ across the Pacific. When vertical wavenumber spectra of vertical strain are nondimensionalized by $\kappa_{0}$, the variability in these pan-Pacific spectra reduce from a factor of 20 to a factor of 2 . Given that numerous dimensionless metrics such as the Richardson number, Froude number, Burger number, etc., are required to specify dynamical balances in the sea, it is intriguing that a single-parameter model describes all aspects of the statistics of vertical strain over the range of scales $\sim 2-200 \mathrm{~m}$. While both internal wave and vortical motions are present in the data, the waves dominate the strain signal at these sites. The high-wavenumber cutoff in the strain spectrum is set by the nonsinusoidal waveform of short-vertical-scale internal waves. As large-scale numerical models improve in resolution, they should replicate this Poisson structure in order to properly model plankton variability, vertical diffusion, horizontal dispersion, sound propagation, and other fine-scale phenomena.
\end{abstract}

KEYWORDS: Inertia-gravity waves; Internal waves; Diapycnal mixing; Microscale processes/variability; Thermocline; Profilers, oceanic

\section{Introduction}

In 1965, Henry Stommel and Konstantin Fedorov (Stommel and Fedorov 1967) were exploring the ocean with a newly developed electronic instrument, "a continuous recorder of salinity and temperature against depth." Following a day of surveying near Timor and a second day off Mindanao, they were impressed that vertical profiles of temperature and salinity in the ocean thermocline are highly irregular, composed of "hundreds of superposed laminae from 2 to $30 \mathrm{~m}$ thick, each fairly homogeneous in temperature and salinity and separated from one another by interfacial regions of much more than average gradient." They mapped out several layers of low vertical gradient, finding them to be of order $10 \mathrm{~km}$ in lateral extent.

Shortly thereafter, Woods (1968) found similar structures off Malta and coined the terms "sheets" and "layers" for distinct regions of high and low vertical gradient. There is now evidence (to be expanded here) that the vertical straining of the thermocline by internal waves leads to much of the irregular structure in the deep open ocean. Small-amplitude waves have sinusoidal waveforms in space and in time. A linear superposition of independent waves leads to Gaussian wavefield statistics. However, the oceanic internal wave field is sufficiently energetic that the vertical waveform of shorter, slower-

¿ Denotes content that is immediately available upon publication as open access.

Corresponding author: Robert Pinkel, rpinkel@ucsd.edu moving waves becomes distorted at depths where vertical propagation speed is inhibited by variations of density gradient or horizontal current. Just as ocean swell steepens and potentially breaks as it shoals, short internal waves steepen when propagating into regions of reduced vertical group velocity. The process was first modeled by Orlanski and Bryan (1969). Desaubies and Gregg (1981) termed wave-induced deformation "reversible finestructure" and demonstrated observationally that Wood's "sheets and layers" were in fact, extreme states of a continuous distribution of vertical gradients.

The irregular structure of the ocean thermocline is receiving renewed attention (e.g., Whalen et al. 2012; Kunze 2017) because the magnitudes of the vertical density gradient spectrum and horizontal shear spectrum have been linked to the mean vertical mixing rate in the ocean, a quantity that is both difficult to directly measure and central to understanding the ocean's role in climate. The Gregg-Henyey (Henyey et al. 1986; Gregg 1989; Polzin et al. 1995) ocean mixing parameterization has been applied to global archives of density profile data with resulting predictions that appear to have considerable skill. The parameterization is interpreted in terms of a weakly nonlinear cascade of energy through the oceanic internal wave spectrum as represented by the Garrett-Munk (GM; Garrett and Munk 1972, 1975; Munk 1981) spectral model.

Thirty years ago, examining an eastern Pacific dataset, Pinkel and Anderson (1992, hereafter PA92; 1997) applied a collection of 10000 CTD profiles $(0-560 \mathrm{~m})$ from the Research Platform (R/P) FLIP to examine the vertical dilation and compression of the thermocline. The statistics of this vertical straining were seen to be almost perfectly Poisson. This finding updated the Hayes et al. (1975) Sargasso Sea observation that 
vertical temperature gradients, the inverse of the PA92 strain, appeared to follow a Poisson distribution.

The many complex processes that affect wave propagation apparently lead to an extremely simple statistical end-state for vertical strain. Is it more efficient to model these processes starting directly from a Poisson perspective, rather than from a linear/Gaussian basic state with nonlinearity added as a perturbation? The situation is somewhat analogous to the problem of molecular diffusion, where a myriad of molecular collisions, very difficult to describe dynamically, are well modeled by a random walk statistical model and characterized by a single diffusion coefficient.

The initial goal of this paper is to determine whether the Poisson behavior seen off the coast of California in 1986 (PA92) is also seen in regions where the dynamical environment is far different. The covariability of fine-scale thermocline straining, the larger-scale internal wave climate, and small-scale overturning is examined using density profile data from seven experiments spanning three decades and much of the Pacific Ocean. Roughly $30000 \mathrm{~km}$ of vertical profile data are here applied to quantify strain in different dynamical settings.

A second objective is to identify the ocean processes that generate these statistics. Can one isolate the contribution of small-scale internal waves to the straining of a thermocline filled with many other types of motion? This is initially done in the depth-time domain. Data are then examined statistically in terms of their variability with background buoyancy frequency. Findings are in general accord with linear internal wave dynamics. Near-seafloor increases in strain variance are seen at sites of topographic generation or dissipation.

\section{Observations}

In 1969 a program was initiated at the Marine Physical Laboratory at Scripps Institution of Oceanography (SIO) to study the depth-time variability of the internal wave field by means of rapid vertical profiling. Observations over a broad range of depth-time scales were required, such that the principal cross-scale linkages in the thermocline could be identified. Initially, high-speed hydraulic winches were installed on the R/P FLIP, enabling temperature profiles of the upper $440 \mathrm{~m}$ of the sea to be collected at 2-min intervals (Pinkel 1975). By the 1983 Mixed Layer Dynamics Experiment (MILDEX), Sea-Bird Electronics SBE9 CTDs were being profiled by electric winches at $3.5 \mathrm{~m} \mathrm{~s}^{-1}$, providing estimates of ocean density with 2-m vertical resolution. In subsequent cruises, Sea Bird SBE9, SBE911, and SBE46 CTDs were profiled singly or in pairs to study open ocean [MILDEX 1983, Patch Experiment (PATCHEX) 1986, Hawaii Ocean-Mixing Experiment (HOME) Farfield 2001], coastal [Applied Research Laboratory (ARL) 1998, Assessing the Effects of Submesoscale Ocean Parameterizations (AESOP) 2006], and intense tidal mixing [HOME Nearfield 2002, Tasman Tidal Dissipation Experiment (TTIDE) 2015] sites. Typical data collection periods spanned 2-4 weeks. Since 2005, this high-speed profiling capability has been transitioned from FLIP to conventional research vessels in support of studies in the western Pacific and Southern Ocean (Fig. 1).
In all cruises, data are obtained at a fixed geographic site, with $\mathrm{R} / \mathrm{P}$ FLIP being anchored in the deep sea or the Research Vessel (R/V) Roger Revelle employing its dynamic positioning system to hold station. The exception is the 1983 MILDEX experiment, where FLIP drifted southward in the California Current as data were collected.

The data collection rates throughout this program are rather larger than the oceanographic norm. In the 2001 HOME Farfield experiment ( $\sim 9500$ profiles to 760 m over 26 days), the profile data rate is roughly $50 \%$ of today's 4000 float global Argo array. While the highly skewed nature of the strain distribution in the thermocline is a robust signal, high-density sampling enables the Poisson probability distribution to be clearly distinguished from other skewed distributions such as the lognormal. Also, a clear view of both thermocline evolution and instrument bias can be seen through the haze of statistical imprecision. A summary of cruise specifics and the data analysis process is presented in Table 1.

Given the high fall rate of the CTDs, it is critical to match the time responses of slower temperature sensors to the more rapid conductivity cells. This is done using cross-spectral techniques, with the resolution of both sensors subsequently limited to $\sim 2 \mathrm{~m}$ by a low-pass filter applied to the response-corrected raw data. In the 2015 TTIDE experiment, a hysteresis correction is added that enables the combined use of both upcast and downcast data. This effectively halves the profile sampling interval from 10 to $5 \mathrm{~min}$ over the depth range $1400-1900 \mathrm{~m}$. For consistency, only the TTIDE downcast data are used in this study.

\section{Quantifying the strain field}

The distortion of the thermocline from its mean state is quantified by tracking the depths $\eta(\bar{z}, t)$ of a family of constant density surfaces that are separated in the mean by a uniform vertical distance $\overline{\Delta z}$. The mean profile is determined by picking an initial set of trial densities $\left\{\rho_{i}\right\}$ and averaging their depths over many independent realizations (vertical profiles) to form $\bar{z}\left(\rho_{i}\right)$, whose inverse $\bar{\rho}(z)$ is termed the semi-Lagrangian (sL) mean profile. This contrasts with the procedure for forming a Eulerian mean, in which density fluctuations are averaged at a series of fixed depths. In a thermocline undergoing Gaussian vertical displacements, sL depth variations observed at fixed density are Gaussian. Density fluctuations observed at fixed Eulerian depths can be significantly skewed in regions where the mean profile has pronounced curvature.

In what follows, the overbar denotes a mean state quantity such as $\overline{N^{2}}(z)$, the undisturbed buoyancy profile, as inferred from a cruise-long average. Brackets \langle\rangle indicate the cruise-long average of a fluctuating quantity. Hatted variables represent quantities with their mean removed, e.g., $\hat{\eta}(\bar{z}, t)=\eta(\bar{z}, t)-$ $\langle\eta(\bar{z}, t)\rangle=\eta(\bar{z}, t)-\bar{z}$. Depth $z$ is taken as positive upward.

Using data from the 1986 PATCHEX in the eastern Pacific, PA92 found that the statistics of the vertical strain, the normalized separation of density surfaces, $\gamma(\bar{z}, t: \overline{\Delta z})=\Delta z(\bar{z}, t) / \overline{\Delta z}$, followed a gamma probability distribution over vertical separation scales $2-64 \mathrm{~m}$. Here, $\Delta z(\bar{z}, t)=\eta\left(\overline{z_{1}}, t\right)-\eta\left(\overline{z_{2}}, t\right)>0$, $\bar{z}=\left(\overline{z_{1}}+\overline{z_{2}}\right) / 2$, and $\overline{\Delta z}=\overline{z_{1}}-\overline{z_{2}}$. 

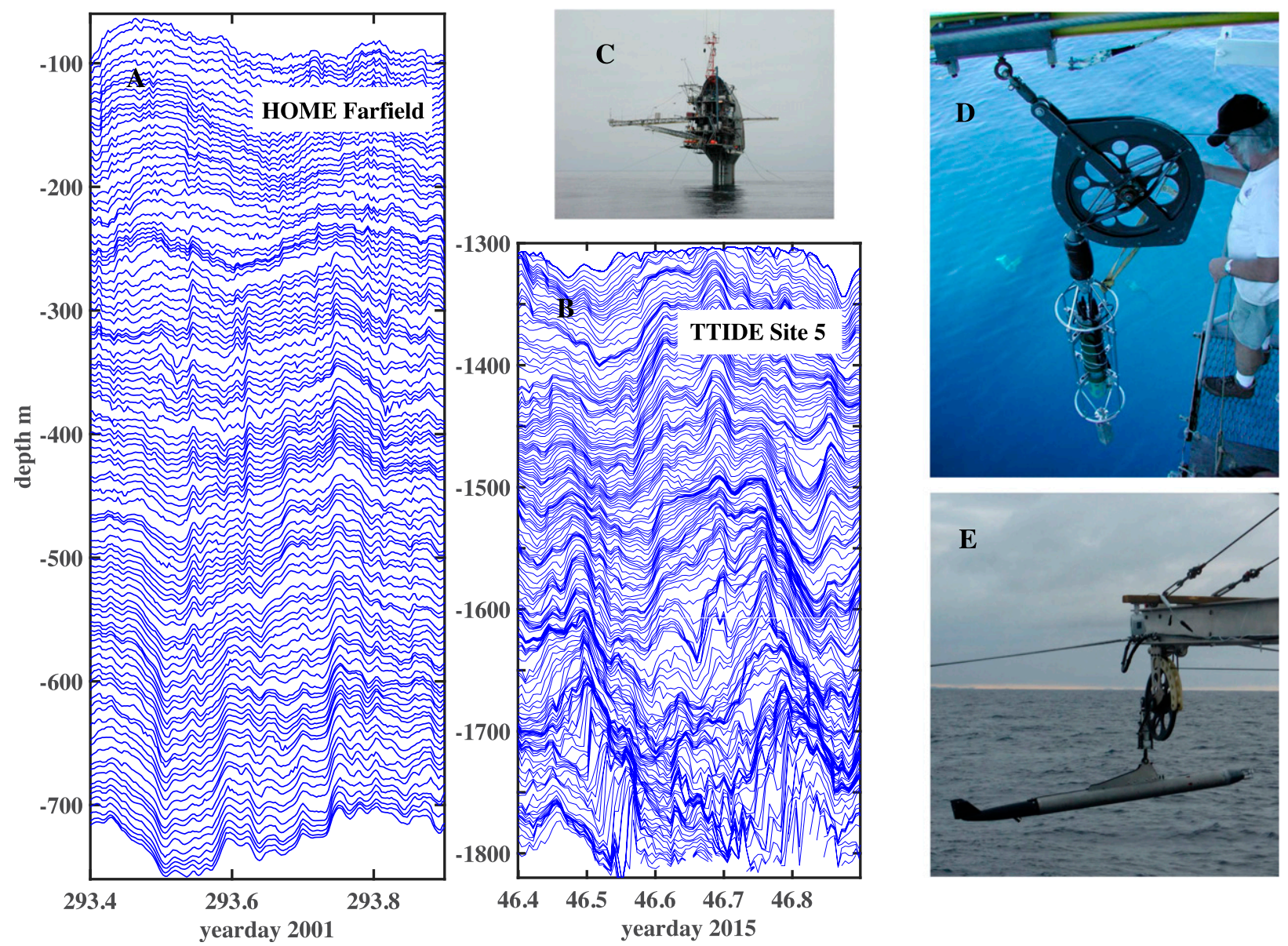

FIG. 1. Data and methods.12-h samples of isopycnal vertical displacement from the (a) HOME Farfield and (b) TTIDE experiments. The Farfield is a typical open Pacific Ocean site. Waves with frequencies above the value of the buoyancy frequency at the sea floor, $\sim 1 \mathrm{cph}$, are low mode with large vertical coherence. The lower-frequency wave field exhibits a broader band of vertical modes. At the TTIDE site, a mode-1 internal tide shoals on the east coast of Tasmania. A 6-h period upslope-propagating first-harmonic of the tide drives an $80-\mathrm{m}$ vertical wall of water along the seafloor, appearing at yearday 46.55 . The fundamental M2 tidal crest arrives at 46.7-46.8, again with bore-like structure at the sea floor. (c) The R/P FLIP hosted the first six experiments described here. (d) Early profiling CTDs were replaced with the up-down sampling (e) Fast-CTD in TTIDE. An acoustic altimeter enabled profiling to within $15 \mathrm{~m}$ of the sea floor in water depths to $2 \mathrm{~km}$.

The gamma distribution is the continuous analog of the Poisson distribution (Papoulis 1984). ${ }^{1}$ Under the constraining definition $\langle\gamma(\bar{z}, t: \overline{\Delta z})\rangle=1$, it is a function of a single parameter:

$$
G^{L}(\gamma \mid \overline{\Delta z})=\frac{\kappa(\kappa \gamma)^{\kappa-1} e^{-\kappa \gamma}}{\Gamma(\kappa)} .
$$

Here, $\kappa=\kappa_{0} \overline{\Delta z}$, where $\kappa_{0}$ is termed the Poisson constant. A sequence of observations from the 2002 HOME Nearfield experiment (Fig. 2) illustrates how the sinusoidal oscillation of surfaces of constant density $\eta(\bar{z}, t)$ in time (Fig. 2a) is completely consistent with their highly nonsinusoidal oscillation in

\footnotetext{
${ }^{1}$ The gamma distribution is more familiar to oceanographers as the $\chi^{2}$ distribution, where it plays a key role in the central limit theorem.
}

depth (Fig. 2b) and highly skewed profiles of surface separation $\gamma(\bar{z}, t: \overline{\Delta z})$ (Fig. 2c).

A comparison between the observed statistics of $\gamma$ and the gamma PDF is presented in Figs. $3 a$ and $3 b$, for the HOME Farfield and Nearfield experiments. The Farfield is taken as representative of a typical open Pacific Ocean environment while the Nearfield is a highly energetic tidal conversion site (Table 1). Agreement with the gamma PDF is excellent over vertical separations $\overline{\Delta z} \geq 4 \mathrm{~m}$. At smaller separations, discrepancies become apparent. In part, these result from the 2-m resolution of the CTD. More fundamentally, interpretation of observations of a Poisson thermocline requires care as one considers vertical scales approaching $\kappa_{0}^{-1}$ and smaller. This important issue is considered in appendix $\mathrm{C}$.

In principle, the single parameter $\kappa_{0}$ governs the evolution of the strain data over all vertical scales $\overline{\Delta z}$. In practice, adjusting $\kappa_{0}$ to provide a best fit to the PDFs at each separation $\overline{\Delta z}$ leads 


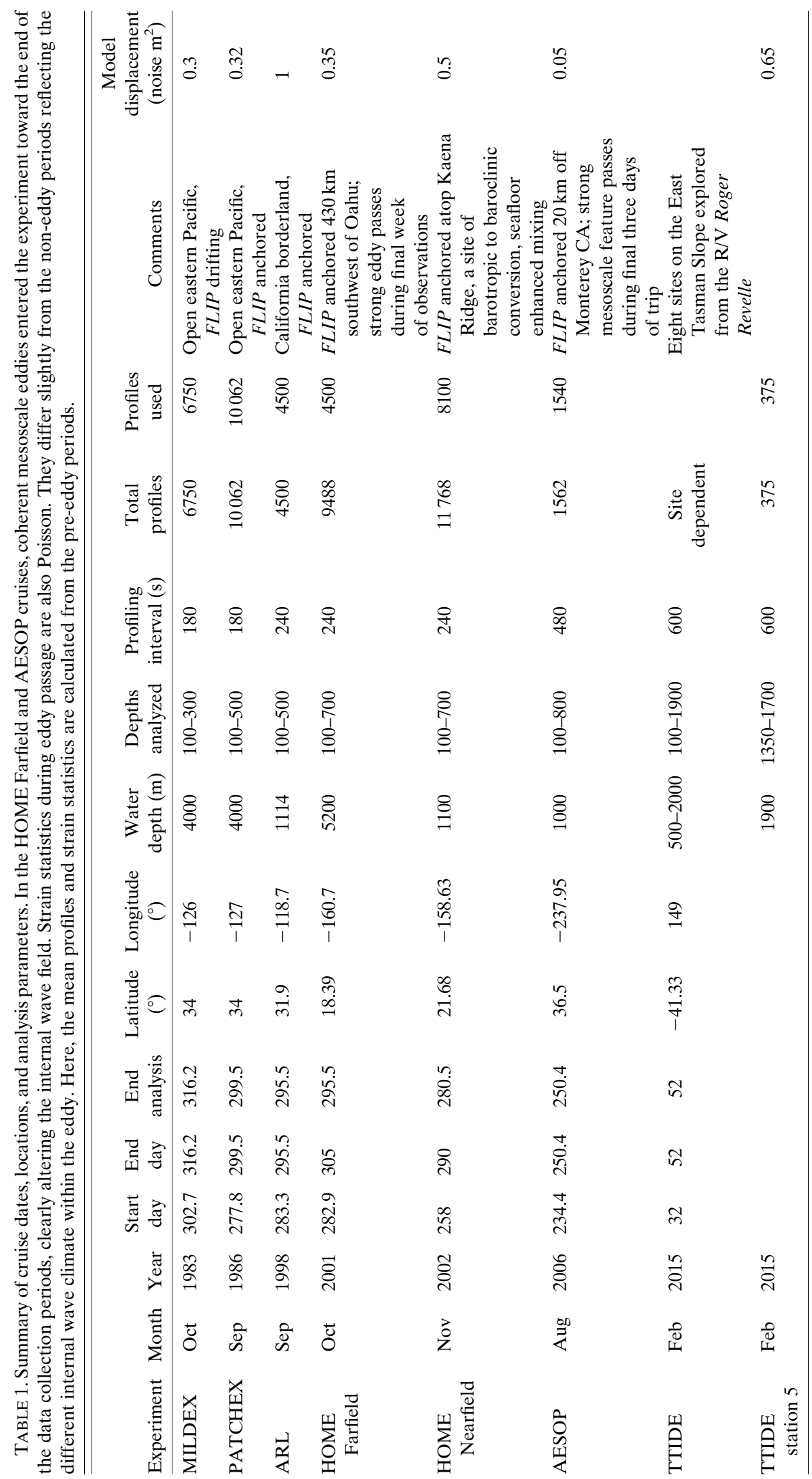



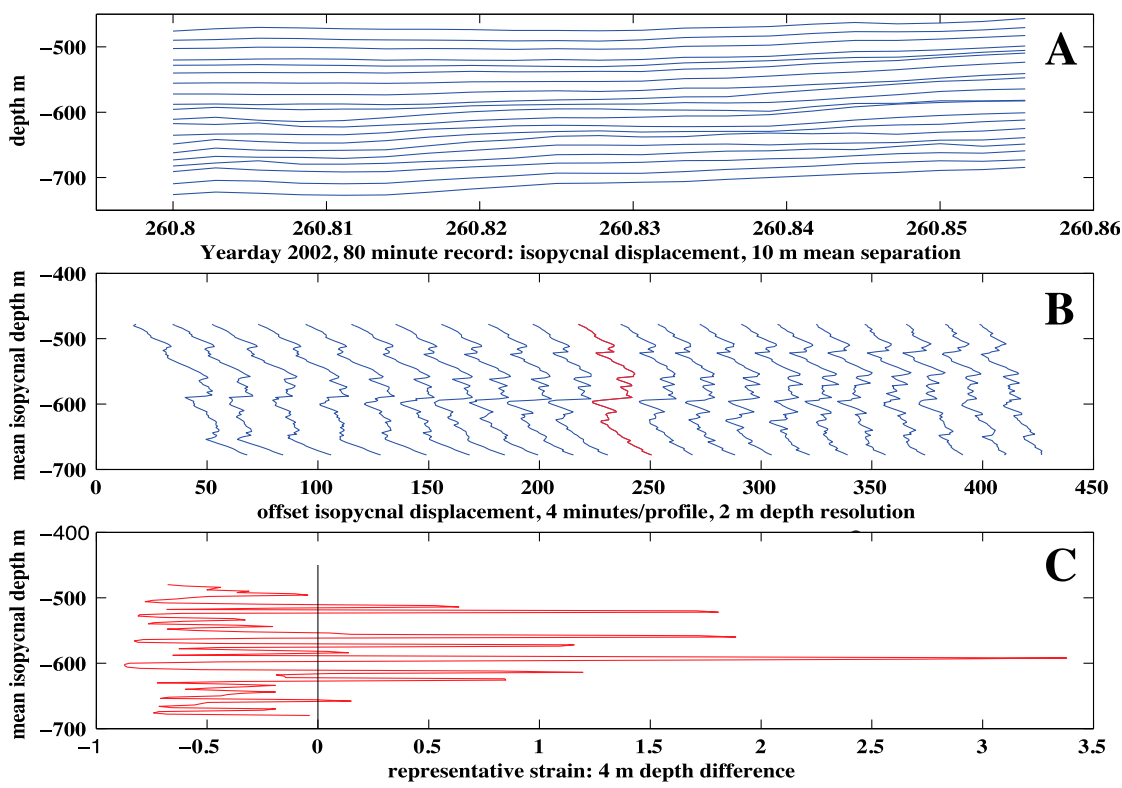

FIG. 2. A close-up of the non-Gaussian strain field in the thermocline. An 80-min, 200-m view of the depth variation of a set of density surfaces from the HOME Nearfield experiment. (a) The vertical displacement of the surfaces varies smoothly in time. (b) However, their variation in depth relative to their time-mean positions is highly irregular. (c) The corresponding 4-m strain, $\gamma-1$, is highly skewed, with most isopycnal pairs being closer together than average in any given realization.

to variations in $\kappa_{0}$ of less than $10 \%$ across a wide range of scales. In Figs. 3a-g, a single value of $\kappa_{0}$ is used to fit all six spatial separations (140 bins each) for each of the experiments, over a single 200-m depth range. The gamma model of these strain PDFs appears equally valid in open-ocean internal wave regimes, in coastal regions, and at intense tidal mixing sites near topography. It will be seen that $\kappa_{0}$ is independent of depth in the open ocean. At sites of strong wavefield bottom enhancement, $\kappa_{0}$ decreases as the seafloor is approached. A summary of observed estimates of the Poisson constant $\kappa_{0}$ is presented in Table 2 for the seven Pacific Ocean sites.

Why is the Poisson-gamma PDF the proper descriptor of thermocline distortion, rather than some other skewed distribution? Consider a set of three density surfaces with adjacent pairs separated in the mean by $\overline{\Delta z}$. The associated finite difference strains are

$$
\gamma_{12}=\left(\eta_{1}-\eta_{2}\right) / \overline{\Delta z}, \quad \gamma_{23}=\left(\eta_{2}-\eta_{3}\right) / \overline{\Delta z} .
$$

If $\gamma_{12}$ and $\gamma_{23}$ fluctuate independently, governed by a probability distribution $P^{L}(\gamma \mid \overline{\Delta z})$ that depends only on parameter $\kappa_{0}$, we require $\gamma_{13}=\gamma_{12}+\gamma_{23}$ to be described by $P^{L}(\gamma \mid 2 \overline{\Delta z})$. This process can be repeated such that $\gamma(n \overline{\Delta z})$ must be described by $P^{L}(\gamma \mid n \overline{\Delta z})$, with the single parameter $\kappa_{0}$ governing the statistical behavior at all separation scales. The gamma PDF is one of a few that maintain this property; most probability families (including the lognormal) do not. The gamma nature of isopycnal separation statistics is completely consistent with the well-known (e.g., Cairns and Williams 1976) Gaussian nature of the displacement field. An explicit representation of the joint probability density of strain and displacement is presented in appendix A and Fig. 3h.

\section{A Poisson statistical model of the thermocline}

In linear wave physics, fluctuations about some background state are usually described in terms of a complete orthogonal set of sinusoidal basis functions. Often there are differences between the physical modes of the process and the sinusoidal analysis modes. Techniques like "WKB stretching" are used to reconcile disparities associated with wave refraction, for example. However, when the physical waveforms are strongly nonsinusoidal, the use of sinusoids as basis functions can confuse the interpretation of field data. For example, the superposition of relatively few nonsinusoidal waves can lead to an apparently continuous broadband spectrum. Efforts to model this situation in terms of a cascade of energy across the apparently continuous spectrum are misguided. Is the thermocline sufficiently distorted that there is value in reconsidering sinusoidal basis functions as the most efficient descriptors?

Here, a discretized Poisson construction of the thermocline is presented. The approach is in the tradition of the random walk diffusion models of classical mechanics. It involves imbedding within the thermocline a vertical stack of "Poisson elements" $\left\{p_{i}\right\}$, each of vertical scale $\kappa_{0}^{-1}$ in the mean (Fig. 4a). Arbitrary mean profiles of scalars such as density or temperature can be imprinted on this stack of elements. In any given realization, the distortion of the mean profile is associated with the random dilation or contraction of these independent Poisson elements (Fig. 4b). The thickness variability of each 

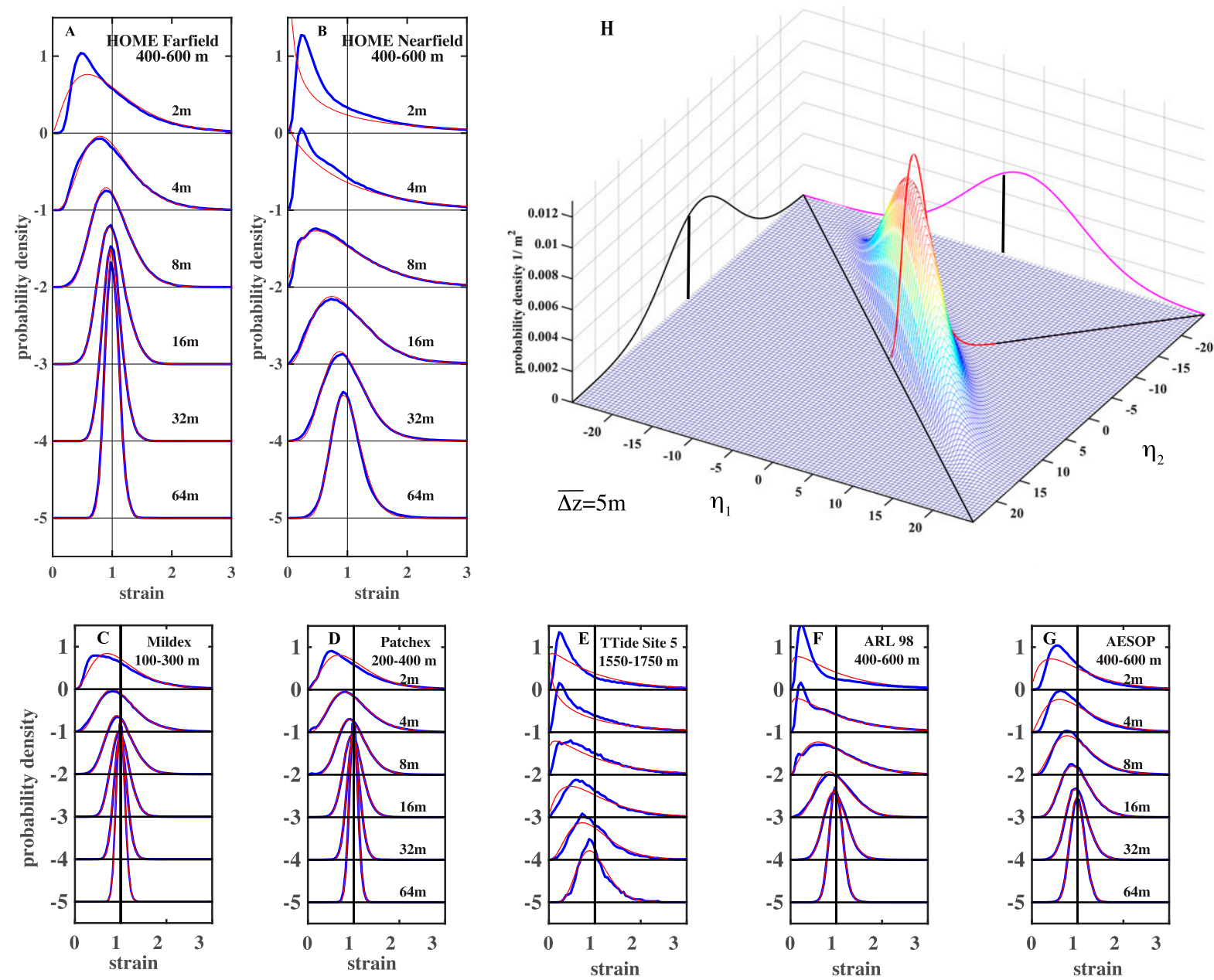

FIG. 3. Probability density functions of strain at mean surface separations of 2, 4, 8, 16, 32, and $64 \mathrm{~m}$ formed from (a) 4500 (Farfield) and (b) 8100 (Nearfield) CTD profiles. Each PDF is estimated in 140 discrete bins over the range $0<\gamma<5$. They thus appear in the figure as continuous curves rather than binned histograms. The observations match the analytical gamma PDF (thin red lines) very well, except at small separations $\overline{\Delta z}$. (a) The low-energy Farfield data are much less skewed than those from (b) the Nearfield at like values of separation. (a)-(g) In examining all Pacific experiments, both the skewness at small separation and the departure from the analytical gamma form increase with increasing 64-m strain variance. (h) A model joint PDF of isopycnal depth and depth difference for isopycnals $\eta_{1}$, $\eta_{2}$, separated by $\overline{\Delta z}=5 \mathrm{~m}$. Here, the isopycnal displacement variance is $50 \mathrm{~m}^{2}$ and $\kappa_{0}=1 \mathrm{~m}^{-1}$. The vertical correlation scale of the displacement field is required to be $Z_{\text {corr_sL }}=2\left\langle\hat{\eta}^{2}\right\rangle \kappa_{0}$ in order that the various projections of the two dimensional PDF replicate the Gaussian PDFs of $\eta_{1}, \eta_{2}$ and the gamma PDF of $\Delta \eta$. Here, the projection of mean displacement (black) is divided by 10 , and the strain projection (red) is divided by 15 . The joint PDF model is presented in appendix A.

element is governed by the exponential PDF, the limiting form of the gamma PDF when $\kappa_{0} \overline{\Delta z}=1$.

Human observers can also impose a grid of reference densities on the sL mean profile and follow these to track thermocline distortion from realization to realization. As a critical aspect of the model, the position of any Poisson element relative to the set of imposed reference densities is both unknown and unknowable. The unknowability condition can be specified by considering a set of four closely spaced (re $\kappa_{0}^{-1}$ ) density surfaces with associated finite difference strains $\gamma_{12}, \gamma_{23}$, and $\gamma_{34}$. Strains $\gamma_{12}$ and $\gamma_{23}$ will be identical from realization to realization if $\rho_{1}, \rho_{2}$, and $\rho_{3}$ fall within the same Poisson element. Partial correlation is possible if $\rho_{2}$ and $\rho_{3}$ span a Poisson boundary. However, in a Poisson process, knowledge of the dependence/independence of $\gamma_{12}$ and $\gamma_{23}$ provides no information relative to the independence of $\gamma_{23}$ and $\gamma_{34}$. This imposes a memoryless constraint on the PDF of the individual Poisson elements and leads to the requirement that their variability be governed by the exponential PDF, $G^{L}\left(\gamma \mid \overline{\Delta z}<\kappa_{0}^{-1}\right)=e^{-\gamma}$. Summing the contributions of adjacent Poisson elements leads to Poisson-gamma behavior.

Synthetic Poisson profiles are easily produced using a random number generator to create exponentially distributed strain realizations $\left\{\gamma_{j}\right\}$ with unity mean and variance. The individual Poisson elements $p_{j}$ that comprise the mean-state thermocline are assigned thickness $\kappa_{0}^{-1} \gamma_{j}$ in each realization. 
TABLE 2. Observed variability in $\kappa_{0}$.

\begin{tabular}{lccc}
\hline \hline & Mean & Standard deviation & $\begin{array}{c}\text { Range (from } \\
\text { shallow to deep) }\end{array}$ \\
\hline MILDEX & 1.58 & - & - \\
PATCHEX & 1.44 & 0.16 & $1.66-1.33$ \\
TTIDE station 5 & 0.26 & 0.15 & $0.41-0.11$ \\
ARL & 0.42 & 0.09 & $0.56-0.38$ \\
HOME Farfield & 1.10 & 0.11 & $1.09-1.14$ \\
HOME Nearfield & 0.29 & 0.09 & $0.44-0.19$ \\
AESOP & 0.49 & 0.05 & $0.55-0.40$ \\
\hline
\end{tabular}

Vertical profiles are achieved by summing adjacent Poisson elements. Representative realizations are presented in Fig. 4c for energetic, $\kappa_{0}=0.5$, through calm, $\kappa_{0}=1.5$, thermoclines. Large-scale "steps" are seen in the energetic profile, with the steps becoming more subtle as $\kappa_{0}$ is increased. If both the depth scale and the vertical gradient are scaled by $\kappa_{0}$ the profiles become indistinguishable (Fig. 4d).

\section{Observing a Poisson thermocline}

Reference isopycnals are human-imposed tracers and have a statistical behavior that differs from that of the underlying
Poisson elements. This results from the inherent uncertainty whether any tracer pair lies within a single Poisson element or spans a Poisson boundary. Modest modifications to the various statistical recipes presented in Table 1 in PA92 and in Table 3 here are required. These modifications become increasingly significant as $\kappa_{0} \overline{\Delta z}$ approaches zero. The gamma PDF itself is defined only for $\kappa_{0} \overline{\Delta z} \geq 1$. A quantitative introduction to the "sampled Poisson" or "microscale" thermocline is presented in appendix $\mathrm{C}$.

In terms of second-order descriptors, the relationship between Poisson elements and human imposed tracers specifies the strain covariance and the vertical wavenumber spectrum. In an sL frame, consider two "point strain" sensors placed in the thermocline at mean separation $\overline{\Delta z}$. The sensors will realize identical strain with unity variance if they happen to fall within the same Poisson element. They will fluctuate independently if their separation spans a Poisson boundary. Thus, the sL strain covariance is just

$$
R_{\gamma}^{L}(\overline{\Delta z})=1-\kappa_{0} \overline{\Delta z}, \quad \kappa_{0} \overline{\Delta z} \leq 1,
$$

where $1-\kappa_{0} \overline{\Delta z}$ is the probability that the two tracers fall within a single Poisson interval of mean thickness $\kappa_{0}^{-1}$. The corresponding sL vertical wavenumber spectrum of strain is
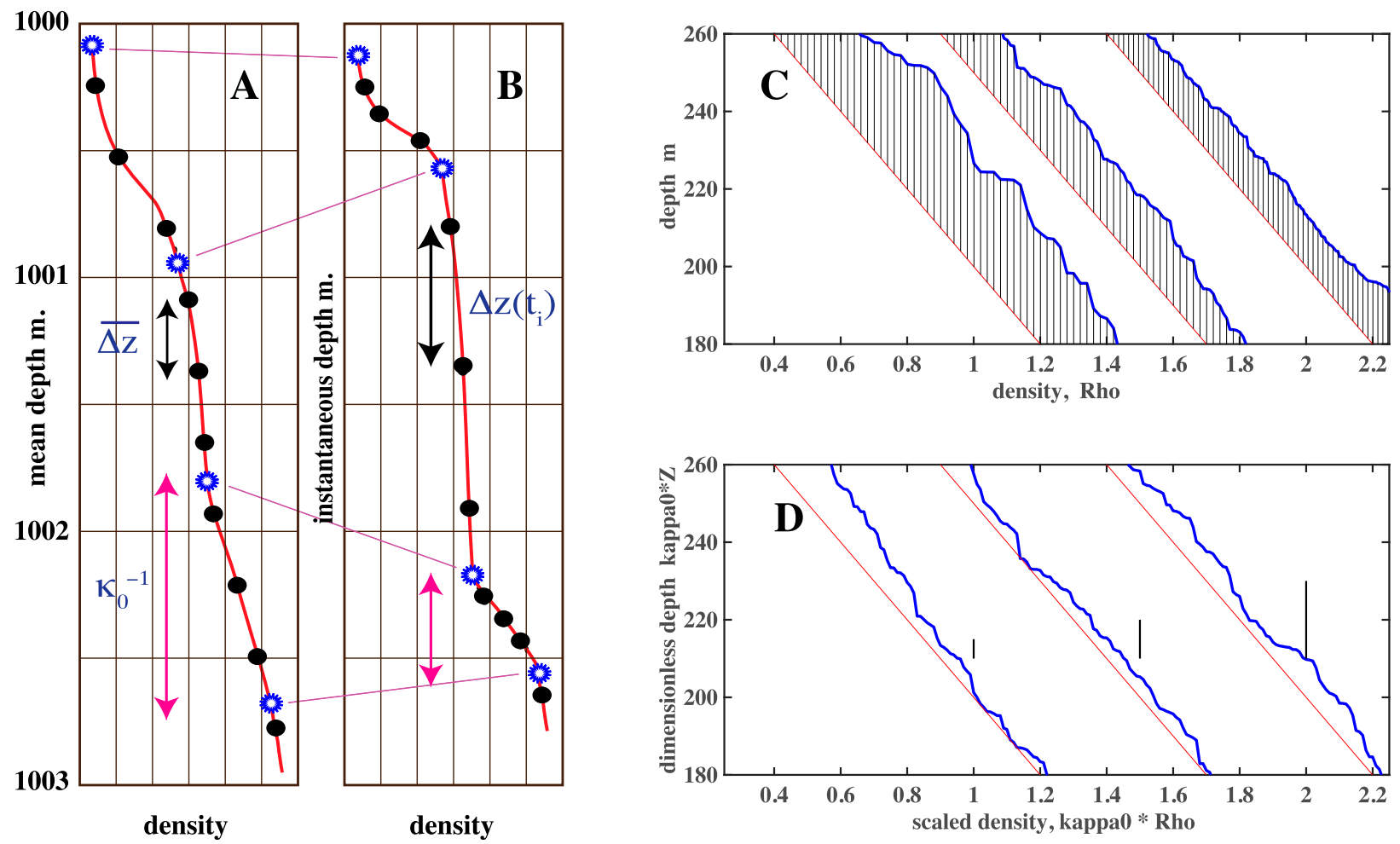

FIG. 4. A schematic Poisson model of thermocline distortion. (a) An arbitrary mean density profile (red) is superposed on an imbedded stack of conceptual Poisson elements, bounded by blue dots, each of mean thickness $\kappa_{0}^{-1} \mathrm{~m}$. A uniformly spaced grid of reference densities (black dots) is superposed. (b) A realization of the distorted profile. The strain within each Poisson element is uniform. (c) Representative realizations of thermocline structure are shown for values of $\kappa_{0}=0.5,1,2 \mathrm{~m}^{-1}$. Both the scale and magnitude of thermocline irregularity increase with decreasing $\kappa_{0}$. (d) Rescaling the depth and density scales by $\kappa_{0}$ renders the profiles indistinguishable. Vertical bars in (d) indicate a 10-m depth interval in the unstretched frame. 
TABLE 3. Poisson summary. Statistical definitions and moments for the idealized Poisson model, neglecting microscale effects; $\kappa \equiv \kappa_{0} \overline{\Delta z}, \gamma \equiv \Delta z / \overline{\Delta z}$, and $\mu=1 / \gamma$.

\begin{tabular}{ll}
\hline \hline Semi-Lagrangian strain & \multicolumn{1}{c}{ Eulerian gradient } \\
\hline$G^{L}(\gamma \mid \overline{\Delta z})=\frac{\kappa(\kappa \gamma)^{\kappa-1} e^{-\kappa \gamma}}{\Gamma(\kappa)}$ & $g^{E}(\mu \mid \overline{\Delta z})=\frac{(\kappa / \mu)^{\kappa+1} e^{-\kappa / \mu}}{\mu \Gamma(\kappa+1)}$ \\
$\langle\gamma\rangle_{\mathrm{sL}}=1$ & $\langle\mu\rangle_{E}=1$ \\
$\left\langle\gamma^{2}\right\rangle_{\mathrm{sL}}=(\kappa+1) / \kappa$ & $\left\langle\mu^{2}\right\rangle_{E}=\kappa /(\kappa-1)$ \\
$\operatorname{Var}_{\mathrm{sL}}(\gamma)=1 / \kappa$ & $\operatorname{Var}_{-E}(\mu)=1 /(\kappa-1)$ \\
$\left\langle\gamma^{3}\right\rangle_{\mathrm{sL}}=\left(\kappa^{2}+3 \kappa+2\right) / \kappa^{2}$ & $\left\langle\mu^{3}\right\rangle_{E}=\kappa^{2} /[(\kappa-2)(\kappa-1)]$ \\
$\left\langle\left(\gamma-\langle\gamma\rangle_{\mathrm{sL}}\right)^{3}\right\rangle_{\mathrm{sL}}=2 / \kappa^{2}$ & $\left\langle\left(\mu-\langle\mu\rangle_{E}\right)^{3}\right\rangle_{E}=4 /[(\kappa-1)(\kappa-2)]$ \\
Skew $_{-}(\gamma)=2 / \sqrt{\kappa}$ & $\operatorname{Skew}_{-}(\mu)=4 \sqrt{\kappa-1} /(\kappa-2)$ \\
\hline
\end{tabular}

$$
S_{\gamma}^{L}(k)=\kappa_{0}^{-1} \sin \left(\pi k / \kappa_{0}\right)^{2} /\left(\pi k / \kappa_{0}\right)^{2}=\kappa_{0}^{-1} \operatorname{Sinc}\left(k / \kappa_{0}\right)^{2} .
$$

Eulerian frame observations differ from their sL equivalents in that the locations of human imposed references, such as fixed depths, vary relative to Poisson boundaries from realization to realization. From Eqs. (7) and (8) in PA92, the exact Eulerian strain covariance is

$$
R_{\gamma}^{E}(\Delta z)=\frac{2}{\left(2+\kappa_{0} \Delta z\right)^{2}} e^{-\kappa_{0} \Delta z}
$$

with corresponding spectrum

$$
S_{\gamma}^{E}(k)=4 \kappa_{0}^{-1} \operatorname{Re}\left\{e^{2\left(1+2 \pi i k / k_{o}\right)} E_{2}\left[2\left(1+2 \pi i k / k_{0}\right)\right]\right\} .
$$

Here, $E_{2}$ is the exponential integral function.

The Poisson strain variance is always unity in the $\mathrm{sL}$ frame and always 0.5 in an Eulerian frame, independent of $\kappa_{0}$. The inability to replicate an internal calm is a potentially disturbing aspect of the model. But this inability is common to many models, including the GM spectral model of the internal wave field (Munk 1981). The issue is associated with model wavenumber spectra that extend to infinitely large vertical wavenumber. In the GM model, for example, the $k^{-1}$ spectral vertical slope at wavenumbers above 0.1 cpm implies an infinite strain variance, independent of the level of the spectrum. To recover finite strain and shear variance, the imposition of a 1-m cutoff is required in the model spectrum. Imposing an analogous high-wavenumber cutoff in the Poisson model leads to a more standard relationship between $\kappa_{0}^{-1}$ and strain variance. We demonstrate in Pinkel (2020, hereafter Part II) that the Poisson wavenumber spectrum can be easily incorporated into a reasonable spectral model for the internal wave field such as the Garrett and Munk (1975) or Munk (1981) models at large vertical scales.

The discrepancy between sL and Eulerian strain variance is inherent in the non-Gaussian nature of the thermocline. Any given Eulerian reference depth spends more time in low-gradient layers than in high-gradient sheets. In turn, reference isopycnals are more often found in sheets than in bounding layers. Similar issues are found throughout fluid mechanics. When working in stream coordinates, for example, averages are colored by the fact that streamlines congregate in regions of high velocity.

Perhaps fortunately, the deformation of the thermocline in an Eulerian frame is not usually quantified in terms of isopycnal separation, but rather by the departure of the local density gradient from the large (vertical) scale mean. If a time average of gradient fluctuations, $\sim 1 / \Delta z(t)$, is formed in an Eulerian frame, the resulting mean gradient is just the inverse of a time average of $\Delta z(t)$ formed in the sL frame, $1 / \overline{\Delta z}$ (PA92). The Eulerian variance of scalar vertical gradients, normalized by the mean gradient squared, is thus identical to the sL variance of isopycnal separation normalized by mean separation squared. This removes the discrepancy between the sL and Eulerian metrics commonly used in oceanography. A model for the vertical wavenumber spectrum of Eulerian vertical gradients is given in appendix $\mathrm{C}$, with model results presented in Fig. 5. In both frames, the Poisson model strain wavenumber spectra are scaled in amplitude as well as wavenumber by $\kappa_{0}^{-1}$. Multiplying these Poisson model spectra by $\kappa_{0}$ and plotting versus dimensionless wavenumber $k / \kappa_{0}$ collapses them to universal forms.

Do oceanic strain spectra share this property? Using data from the seven pan-Pacific cruises, strain records from successive 200-m depth intervals, overlapping by $100 \mathrm{~m}$, are Fourier transformed in the vertical. Strain spectral estimates are formed and averaged over 200 (TTIDE) to 7000 (HOME Nearfield) successive profiles. The spectra are corrected for sensor resolution and for the finite-difference approximation used in converting discrete vertical displacement records to strain. A $k^{2}$ noise spectrum is removed from each estimate using the noise variance (Table 1) based on the observed precision in estimating the depth of density surfaces. The plots are terminated at high wavenumber when the corrected spectral level approaches the level of the noise correction (Fig. 6a), typically at 5-10-m vertical scale.

Estimates of $\kappa_{0}$ are required to implement the Poissonscaling of the spectra. Here these are formed based on averages of the mean cube of vertical strain over the depth-time intervals used for the spectral estimates. Pairs of density surfaces separated by $\overline{\Delta z}=8 \mathrm{~m}$ are tracked and estimates of $\kappa_{0}$ are determined from the Poisson-derived relations in Table 3 as modified in appendix C, Eq. (C7b).

The data span a range of environments from typical midocean to coastal, including regions of intense near-seafloor breaking waves. The corresponding unscaled spectral levels vary by a factor of 20 at low wavenumber, as do the values of $\kappa_{0}$ derived from the mean cube of the $8-\mathrm{m}$ strain. When these spectra are normalized by $\kappa_{0}$ and plotted as a function of $k / \kappa_{0}$ the resultant dimensionless spectra collapse to within a factor of 2 (Fig. 6b). The success of this scaling is more apparent when a red form of the spectrum, such as the vertical displacement spectrum $S_{\eta}^{L}(k)$, is considered (Figs. 6c and $6 \mathrm{~d}$ ). These are formed by dividing the strain spectra by $(2 \pi k)^{2}$. The displacement spectra have a classic $k^{-2}$ slope at low wavenumber, steepening at scale smaller than $2 \pi \kappa_{0}^{-1} \mathrm{~m}$. The Poisson normalization results in spectral variability of the width of the plotted lines over four orders of magnitude in displacement variance density. 

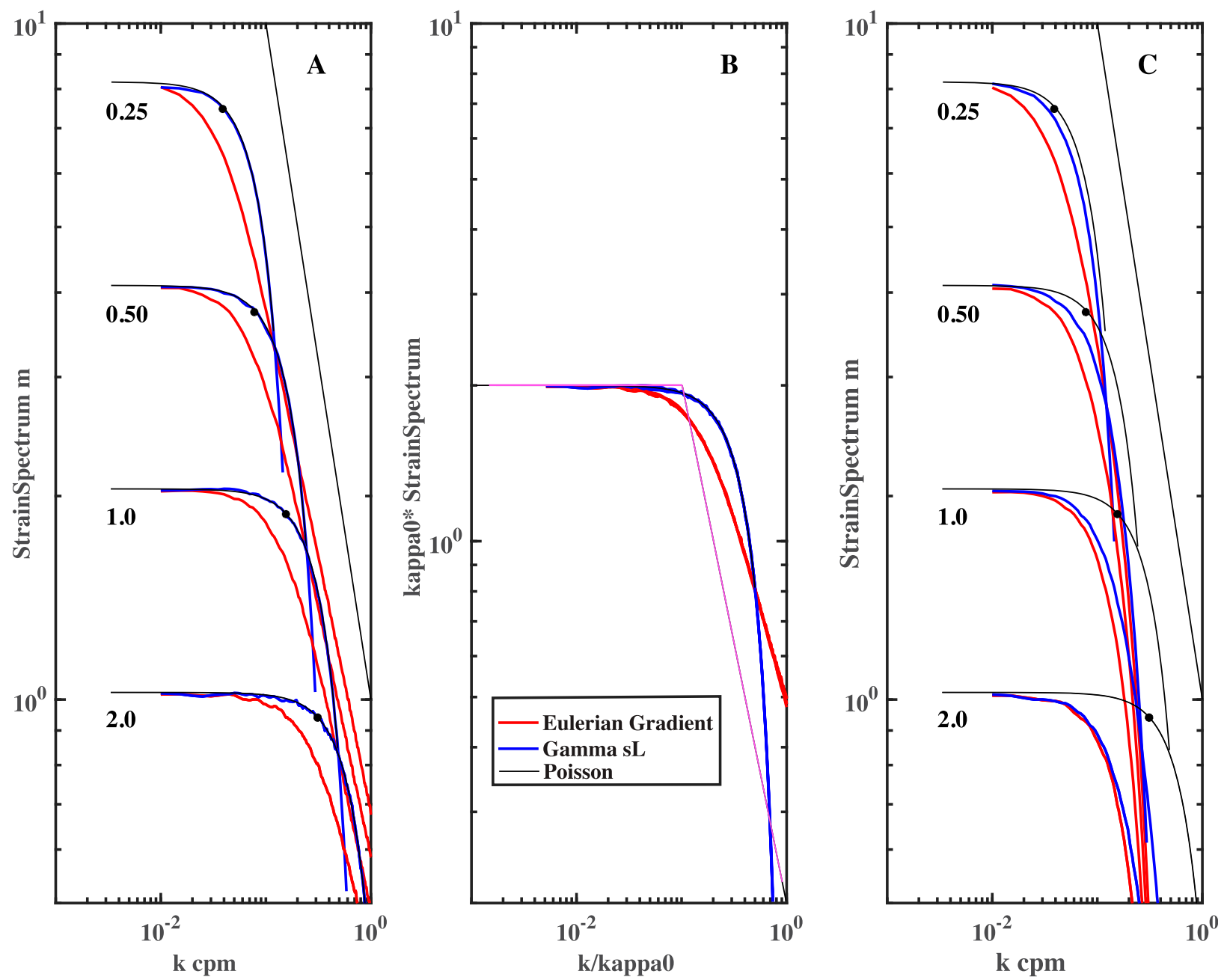

C_2019 E f 5

FIG. 5. (a) Synthetic vertical wavenumber spectra of 1-m strain at four values of $\kappa_{0}$. These are obtained from 20000 realizations of the synthetic profiles in Fig. 4c. The sL spectra (blue) are derived from the vertical displacements of a set of reference densities superimposed arbitrarily on the generating Poisson elements. Corresponding Eulerian spectra of density gradient (red) exhibit a high-wavenumber cutoff that starts at lower wavenumber and decays more slowly than the sL spectra, based on the identical synthetic profiles. (b) In both frames, the spectra collapse to a canonical form when nondimensionalized by $\kappa_{0}$. (c) To simulate finite sensor resolution, the individual profile realizations are smoothed by two meters and a new "low resolution" set of isopycnals is tracked. The low-variance (high $\kappa_{0}$ ) spectra are affected to a greater extent than the high-variance profiles by sensor resolution. The Munk (1981) version of the GM spectral model is given as the magenta line in (b).

Importantly, no consideration of rest-state parameters such as the background density gradient $\left(\overline{N^{2}}\right)$ is involved in this rescaling, even though $\overline{N^{2}}$ varies over two orders of magnitude across the dataset. Traditional internal wave scaling relations based on the WKB approximation (e.g., Garrett and Munk 1972, 1975; Gill 1982) are not invoked.

The power spectrum carries information only about secondorder variability, the variance. By contrast, the mean cube strain at 8-m scale apparently contains all information required to reproduce the spectrum of strain and displacement in the thermocline over vertical scales from $200 \mathrm{~m}$ through the slope break at $\kappa_{0} / 2 \pi \mathrm{cpm}$ to scales of order $\kappa_{0}^{-1}$. In principle, estimates of $\kappa_{0}$ can be obtained using any of the moments of strain obtained at any scale. The use of the third moment here emphasizes the asymmetry in information content in the spectral versus Poisson descriptions of the thermocline.

\section{What strains the thermocline?}

It is somewhat disturbing to demonstrate a predictive capability that requires so little specification of the processes involved. A close examination of thermocline variability in depth and time is appropriate, with the objective of identifying the phenomena that establish the strain field. As representative of the diverse sites, observations from the open-ocean HOME Farfield Experiment and the California coastal AESOP 

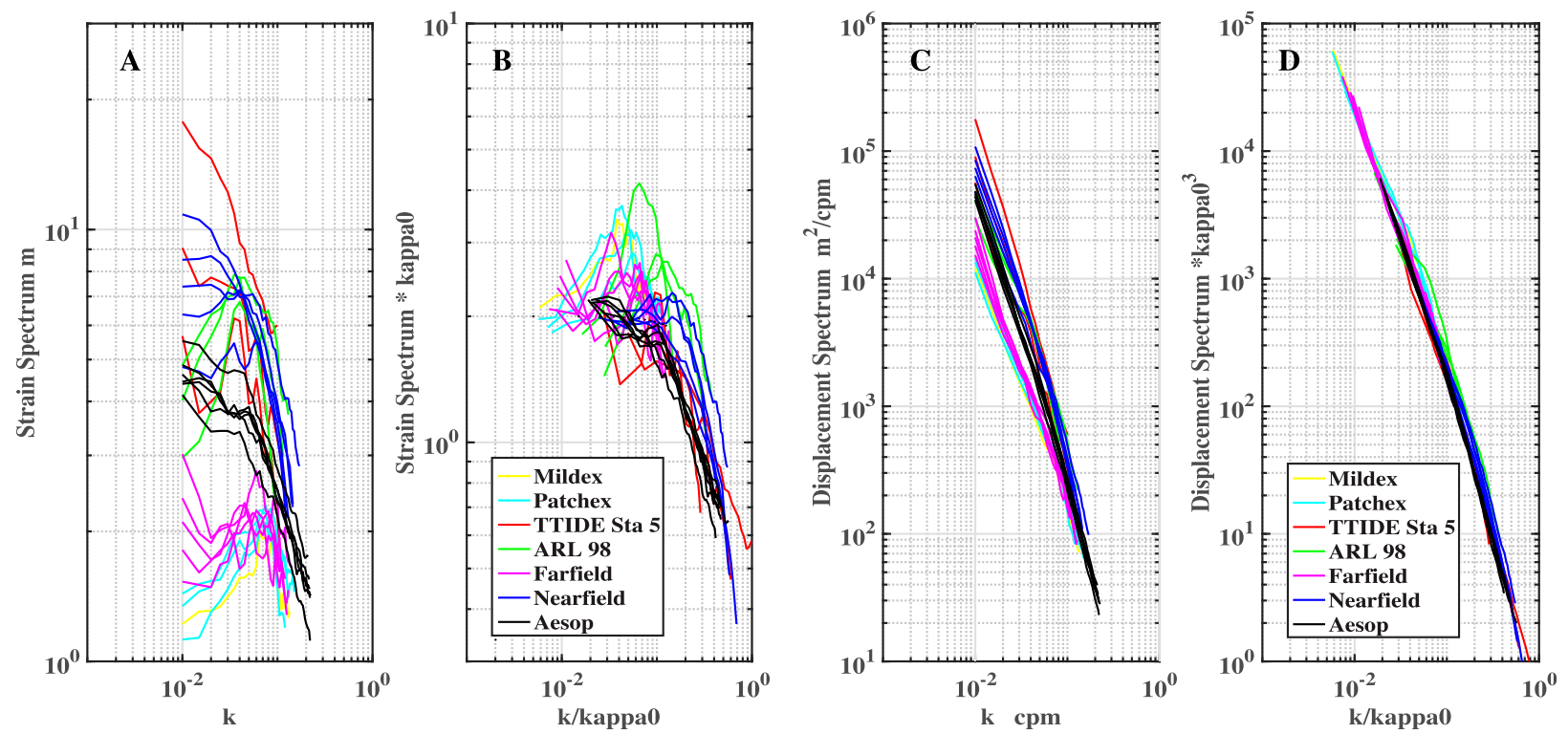

FIG. 6. Vertical wavenumber spectra of strain and displacement from the seven field programs. Like-colored spectral estimates correspond to different 200-m depth intervals, starting below $200 \mathrm{~m}$ and overlapped by $100 \mathrm{~m}$. Typically, 2000-9000 profiles are used in each estimate, with the exception of the TTIDE experiment. (a) Strain and (c) corresponding vertical displacement spectra demonstrate pan-Pacific variability over the 30-yr collection period. (b),(d) The associated dimensionless strain and displacement spectra are rescaled by values of $\kappa_{0}$ determined from estimates of 8-m strain skewness in each of the depth ranges. All spectra collapse to within a factor of 2 under this scaling.

experiment are presented. The HOME data were collected approximately $450 \mathrm{~km}$ southwest of the island of Oahu (Table 1) over a 4-week period in fall 2001. A 9600 profile record (0-760 $\mathrm{m})$ from profiling CTDs is here complemented by horizontal velocity data from the SIO Deep-8 Doppler sonar, an eight-beam sonar that transmitted broadband coded pulses both upward $(160 \mathrm{kHz})$ and downward $(140 \mathrm{kHz})$ from its 380-m operating depth. The nominal vertical resolution of the sonar, as determined by a combination of transmitted pulse duration and processing smoothing, is quantified by a triangular smoothing function of $4 \mathrm{~m}$ width at half-height.

A close-up of the vertical displacement of the density field is given in Fig. 7a. Density surfaces are indicated by black lines and the separation between surfaces is given by the background color. Large positive strains (layers, yellow) are associated with crests of the dominant semidiurnal internal tide and also with thinner layers that appear to ride on the density surfaces. These identical data are plotted in a sL coordinate system (Fig. 7b). Here the ordinate specifies the mean depth of the density surface being tracked. Large positive strains now appear as yellow lines that fall in two general classes. Those layers that are fixed to density surfaces appear as horizontal lines. They have a typical lifetime of $8-15 \mathrm{~h}, 1 / 4-1 / 2$ inertial period at the site. A second grouping of layers appears to propagate across density surfaces and is associated with small-scale internal waves.

The signature of the nonpropagating strain signals, thought to be associated with small-scale quasigeostrophic (vortical) motions, can be removed from the record by a high-pass filter that operates in time in the $\mathrm{sL}$ frame. The residual signal (Fig. 7d) gives the strain field of vertically propagating waves. Small-scale wave phases, as indicated by regions of positive strain, propagate vertically at speeds of $50-150 \mathrm{~m} \mathrm{day}^{-1}$. There appears to be more small-scale activity when a large-scale internal tide or near-inertial wave dilates the overall thermocline (lighter regions), reducing the large-scale buoyancy frequency.

The vertical propagation of a short internal wave is strongly affected by the horizontal background current through which it propagates (e.g., Bretherton 1966). In Figs. 7c, 7e, and 7f, the magnitudes of the absolute current and the zonal and meridional shear are presented, again in a reference system that moves vertically with the density field. Black vertical lines give the depth-time variability of strain, with positive strains corresponding to a rightward offset.

The dominant shear at the Farfield site is associated with near inertial waves. This large-scale shear pattern propagates vertically at speeds comparable to that of the small-scale waves (Broutman 1984). The response of a given wave packet to current variability depends on the packet's horizontal phase speed and azimuth of propagation. Strong strain responses can be seen associated with both positive and negative shear features in the zonal and meridional records. The near-inertial velocity field also advects these small packets laterally, limiting the viewing time of packet propagation.

To augment the open ocean view from the Farfield, the strain field from the 2006 AESOP experiment is presented in Fig. 8. AESOP is located in 1-km deep water approximately $20 \mathrm{~km}$ west of Monterey, California. Relative to the other Pacific sites considered here, AESOP features an internal wave field of typical energy that is interacting with the irregular sea floor below. The submesoscale is more energetic than in the open ocean, with pronounced variability in $T-S$ structure that provides a useful signature of lateral advection. The AESOP strain field is plotted 


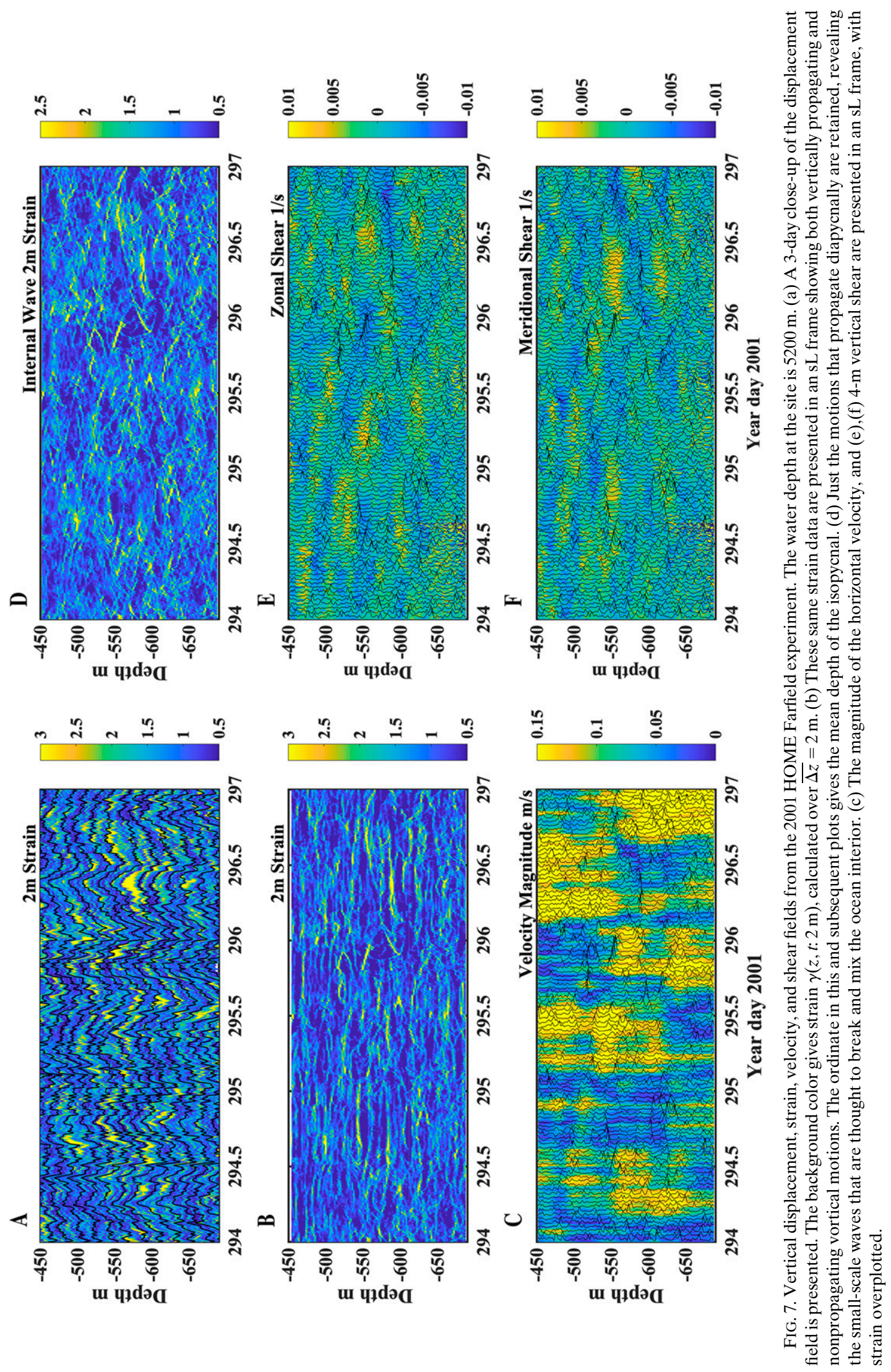




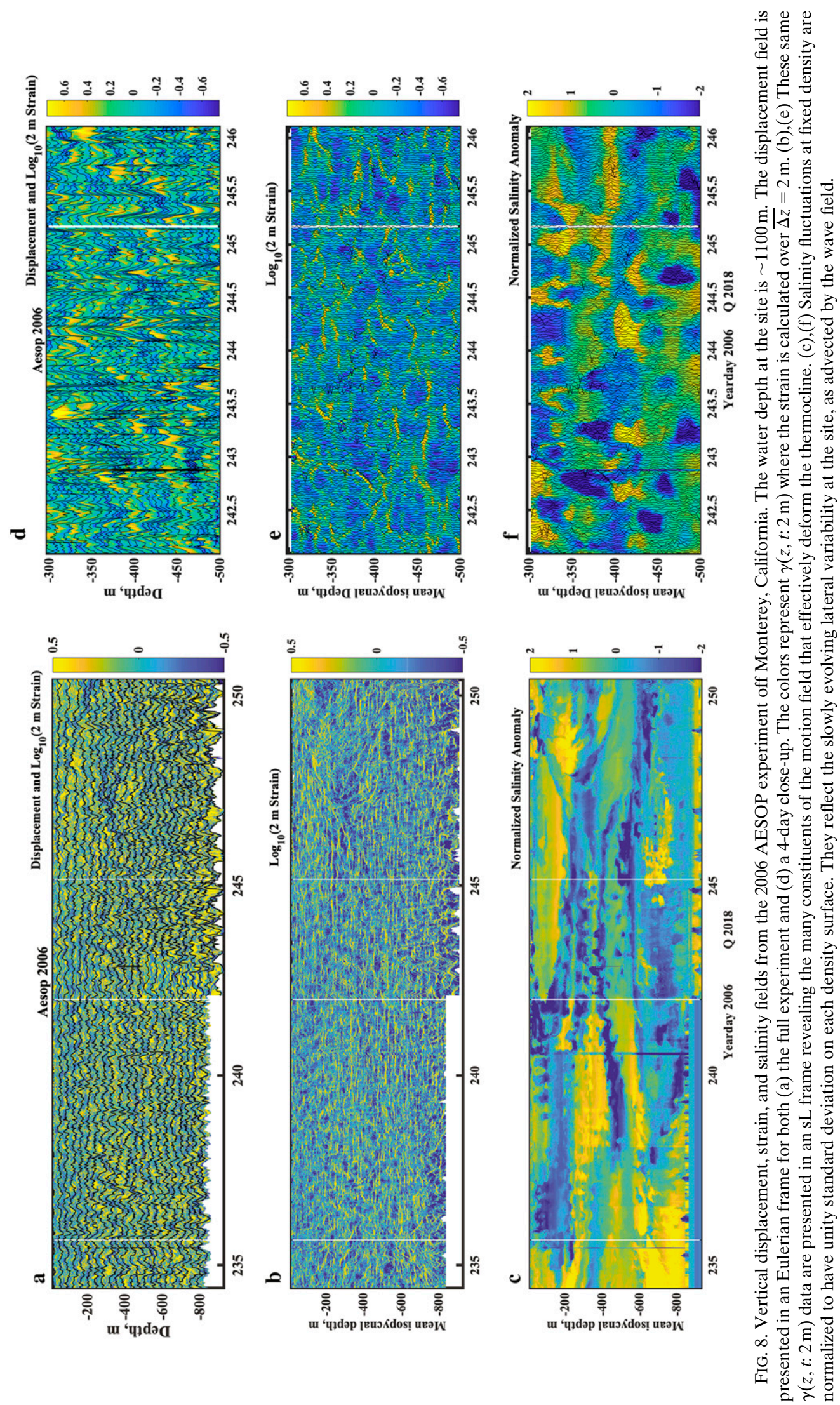



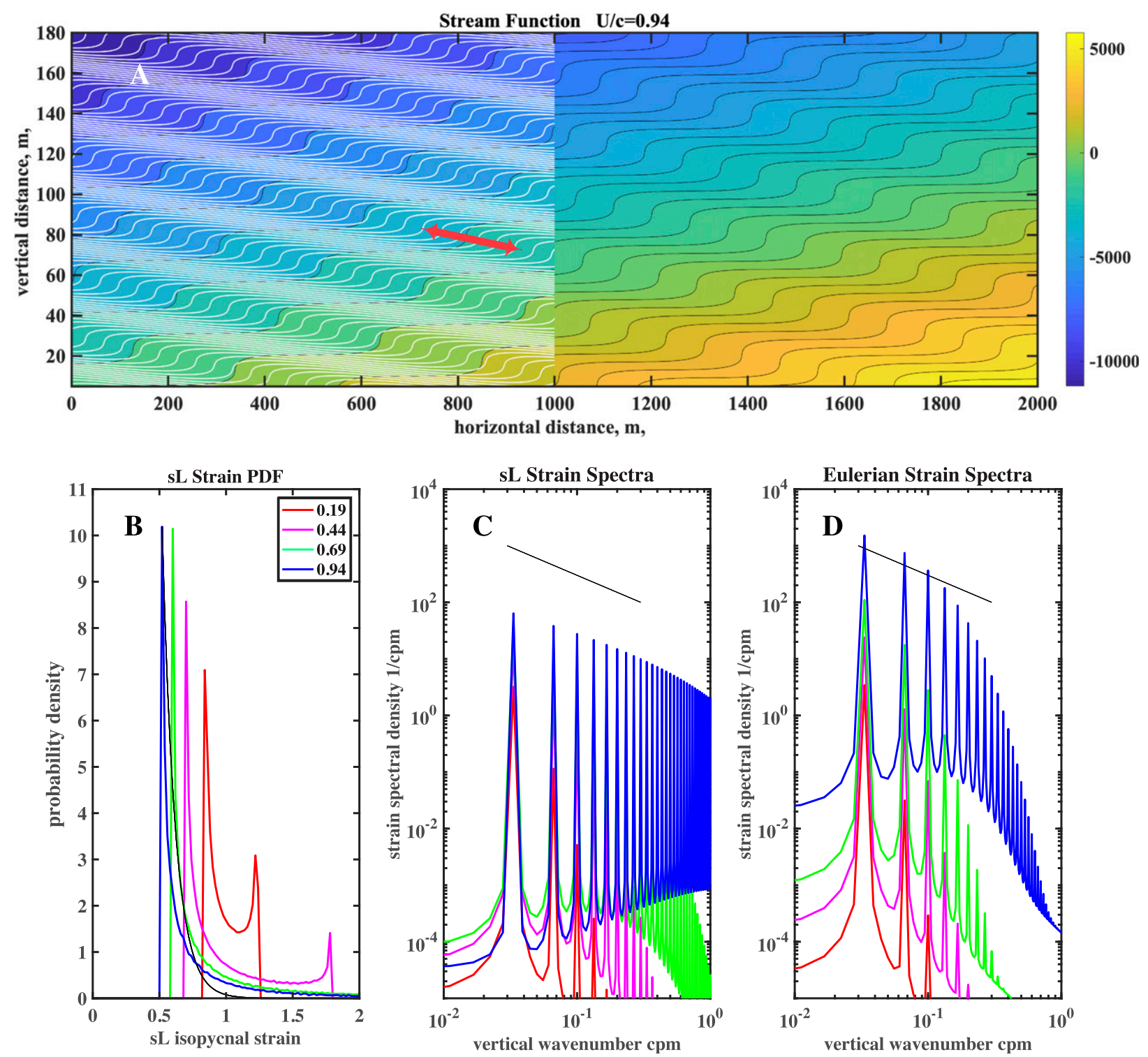

FIG. 9. (a) Nonlinear model representation of a large-amplitude plane wave propagating in the positive $x$, $z$ directions. Colors give contours of the streamfunction of the flow, as seen in a reference frame moving in the positive $x$ and $z$ directions with the wave phase speed. Representative isopycnal surfaces are indicated in white in the left half of the figure. The red arrow indicates the direction of wave displacement perturbations. (b) The PDF of strain is given for four values of the nonlinearity parameter $U / c_{x}$ for semi-Lagrangian frame averages. An exponential PDF matched to the peak of the $0.94 U / c_{x}$ model PDF is plotted in black for reference. (c),(d) Vertical wavenumber spectra of strain for the four values of $U / c_{x}$ are given for $\mathrm{sL}$ and Eulerian frames. The fundamental wave has a purely sinusoidal velocity field with a vertical wavelength of $30 \mathrm{~m}$. The high wavenumber " $k_{z}^{-1}$ subrange" (black reference line) results from the distortion of the density field by this sinusoidal velocity.

in Figs. 8a-c, with data smoothed by a 2D Gaussian filter of width $20 \mathrm{~m}$ in depth and $4 \mathrm{~h}$ in time. The Eulerian 2-m strain (Fig. 8a) is punctuated by layers (yellow) that are modulated by vertically propagating tidal internal waves. A few subinertial layers (e.g., $380 \mathrm{~m}$, yearday 239-241) stand above the internal wave background. Starting at day 246 a mesoscale feature advects into view, occupying progressively more of the water columns.

These same data are presented in an sL frame (Fig. 8b). Positively strained (yellow) layers are vertically compacted in the sL frame while negatively strained sheets (blue) expand to fill the density domain. Again, the modulation of the vertically propagating tide is apparent at largest scales. In other Pacific datasets, where vertical propagation of the tide is less pronounced, slightly superinertial high-mode waves are the dominant source of large-scale, low-frequency strain.

How does the strain relate to the local variability in $T-S$ structure? To visualize this, we plot the variability of salinity on density surfaces (Fig. 8c). The temporal mean is removed from 
the salinity signal on each density surface and the fluctuating residual signal is normalized to have unity standard deviation. This salinity anomaly signal shows slow variations on a time scale of $\sim 1$ week and depth scales of $100-300 \mathrm{~m}$. The images are beautifully modulated by the internal tide which is rhythmically advecting lateral variability along the density surfaces under FLIP. This is a rare field example of internal waves being inefficient, relative to balanced motions, at "stirring" scalar fields on isopycnal surfaces. The arrival of the eddy, an efficient stirrer, on yearday 246 brings new, nonrepetitive salinity anomalies into view.

To explore these patterns more closely, a 3.5-day 200-m section of the strain field is presented in Eulerian and sL frames (Figs. 8d,e) along with the corresponding sL salinity anomaly (Fig. 8f). Here data are smoothed with a 2D Gaussian window of width $3 \mathrm{~m}$ in depth and $38 \mathrm{~min}$ in time. Again, one sees layers (yellow, Figs. 8d,e) appearing with tidal modulation and moving vertically across the density field at the vertical phase speed of the tide. More subtle patterns appear in the sL frame (Fig. 8d) with indications of both upward and downward propagation. The suggestion is that the sloping strain features that are seen to cross density surfaces are in fact short gravity waves, whose vertical propagation is modulated by variation in the background velocity or strain fields in either depth or time. The most visible packets are the ones whose propagation has been impeded at the depth of observation. Their energy density is large as a consequence of the local convergence of the vertical energy flux. These waves have near-zero intrinsic frequency, which is synonymous with the statement that they are "imbedded in the background" flow. Their apparent vertical propagation is in part due to the lateral advection of sloping features. Temperature-salinity anomalies are not associated with their passage.

\section{The strain field of a single nonlinear plane wave}

Does a nonlinear internal wave distort the thermocline in a manner consistent with the observations? It is instructive to generate an analytic internal wave and investigate its strain as a function of the degree of nonlinearity. While such studies are usually pursued numerically, a plane wave of infinite extent is easily generated following Phillips (1966) observation that a sinusoid is an exact solution of the inviscid Navier-Stokes equations in a fluid with constant buoyancy frequency N. Neglecting Earth's rotation, a two-dimensional wave of frequency $\sigma$, wavenumbers $k_{x}, k_{z}=k_{x}\left(N^{2} / \sigma^{2}-1\right)^{1 / 2}$ can be described by the streamfunction

$$
\psi(x, z)=\frac{U}{2 \pi k_{z}} \sin \left[2 \pi\left(k_{x} x+k_{z} z\right)\right]-\left(c_{x} z-c_{z} x\right) .
$$

Here, $c_{x} \equiv \sigma / k_{x}, c_{z} \equiv \sigma / k_{z}$, and the streamfunction is given in a reference frame moving at the phase speed of the wave, such that time dependence vanishes. Spatial variability in velocity is given by the appropriate derivative of the streamfunction, with $U$ specifying the magnitude of the horizontal orbital velocity. In this time-independent frame, flow streamlines are congruent with water parcel trajectories and the strain field can be inferred directly from the velocity field. Thus, normalized vertical strain can be defined as

$$
\gamma=\Delta z(x, z) / \overline{\Delta z}=[(\partial \psi / \partial z) /\langle\partial \psi / \partial z\rangle]^{-1}=c_{x} /\left[c_{x}-u(x, z)\right] .
$$

Eulerian averages of strain and inverse strain $\gamma^{-1}=N^{2} / \overline{N^{2}}=$ $1-u(x, z) / c_{x}$ can be formed directly from (6). Importantly, the model Eulerian strain field becomes highly distorted as $U \rightarrow c_{x}$, whereas the Eulerian inverse strain $\gamma^{-1}$ remains sinusoidal at all values of nonlinearity.

The distortion of isopycnal surfaces by the passing wave must be determined to model sL frame statistics. Because water parcels travel along streamlines, one can label any parcel uniquely by its $x$ or $z$ position in the undisturbed fluid, and its unchanging value of $\psi_{0}(x, z)$, as determined from (6) in the limit $U=0$. For finite $U$, the map of $\psi(x, z)$ specifies the point of origin of the parcels that have arrived at $(x, z)$ under wave displacement. For plane waves, this displacement is parallel to wave crests and the vector displacement that brings any particle from some $\left(x_{0}, z_{0}\right)$ to $(x, z)$ is identical to the displacement experienced by the particle originally at $(x, z)$. This renders the calculation of the wave displacement field and isopycnal position straightforward.

Example flow fields are calculated for a wave of frequency $\sigma=$ $0.1 \mathrm{cph}, k_{x}=1 / 600.3 \mathrm{cpm}$, and $k_{z}=1 / 30.05 \mathrm{cpm}$ in a thermocline with $N=2 \mathrm{cph}$. Nonlinearity is set by the ratio $U / c_{x}$, which is varied from 0.19 to 0.94 in four realizations of the flow, Fig. 9. In the frame of the wave, moving upward and to the right in Fig. 9a, the streamfunction describes spatial oscillations of a flow with a mean component downward and to the left, $\bar{u}=\left(-c_{x},-c_{z}\right)$.

Eulerian strain statistics can be formed simply by averaging statistics of $c_{x} /\left[c_{x}-u(x, z)\right]$ over the domain of the calculation (Fig. 9a). Semi-Lagrangian averages are formed from isopycnal separation data. The probability density function of $\mathrm{sL}$ strain is given in Fig. $9 \mathrm{~b}$ for four values of $U / c_{x}$. The familiar two-horned probability density of a sinusoid is recovered for small $U / c_{x}$. As wave amplitude is increased, the horns spread and become unequal, with a highly skewed exponential-like density function associated with the most nonlinear example. The PDF is in fact narrower than an exponential of similar range.

Vertical wavenumber spectra of simulated strain are presented in Figs. 9c and 9d. The development of harmonic structures is clearly seen as nonlinearity is increased. In the Eulerian description, the energy density of the fundamental peak at $1 / 30 \mathrm{cpm}$ continues to increase as nonlinearity increases. In the sL view the fundamental peak saturates. Added variance appears primarily in the harmonics.

These spectra are calculated using a synthetic data record of $180 \mathrm{~m}$, such that the $30.05-\mathrm{m}$ fundamental wave appears primarily in the sixth Fourier band. The harmonics similarly fall in discrete Fourier bands. Were the record length arbitrary relative to the wavelength of the fundamental wave, the harmonic "comb" structure would be blurred into a quasi-continuous spectral subrange.

This simulation clearly illustrates that the motion field in the $k_{z}^{-1}$ region of oceanic shear and strain spectra can be understood in terms of forced harmonics of larger-scale waves. 

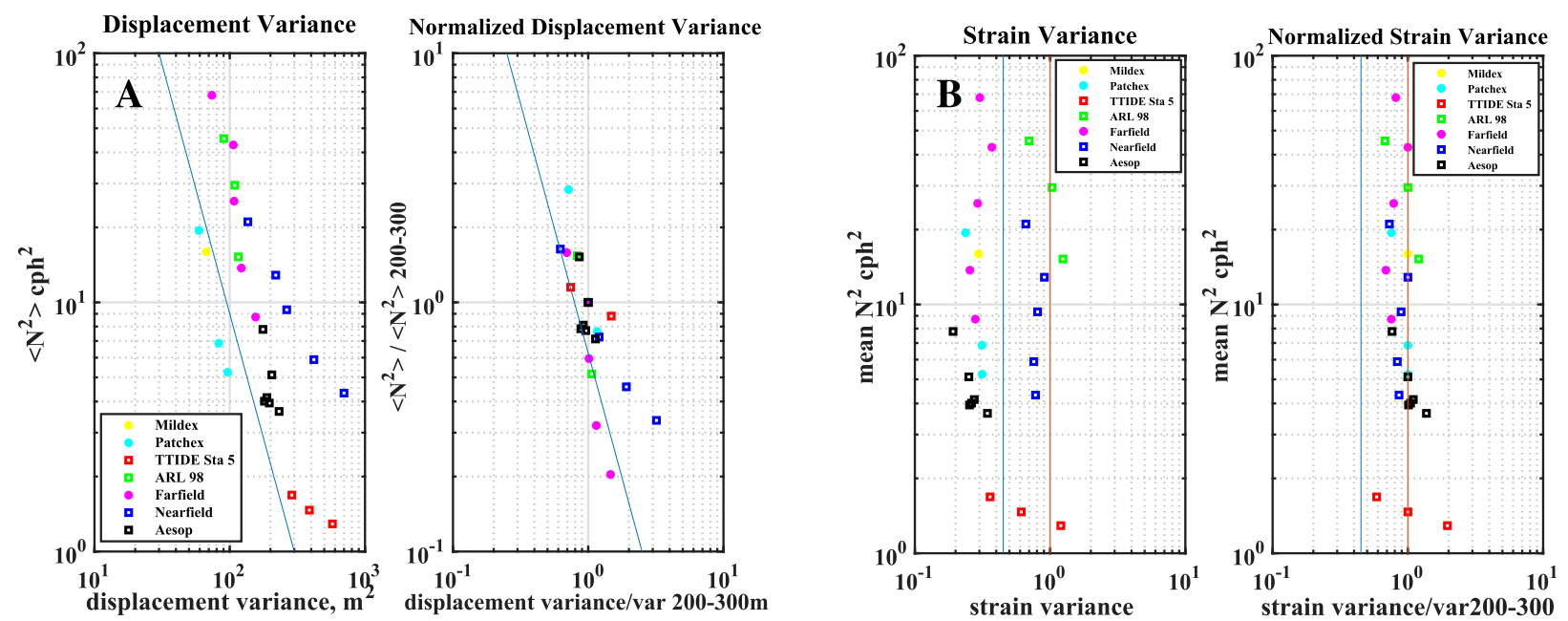

FIG. 10. Vertical displacement and strain variability as a function of $\overline{N^{2}}$ and $\overline{\Delta z}$. (a) The displacement variance of density surfaces (shown at left) generally increases with depth as $\left(\bar{N}^{2}\right)^{-1 / 2}$ at the individual sites, consistent with wave propagation in the WKB approximation. (b) Two-meter strain variance (shown at left) is generally uniform over variations of two orders of magnitude in $\overline{N^{2}}$. To reduce site-to-site variability, mean buoyancy frequency and observed displacement variance are normalized by their $200-300-\mathrm{m}$ values (1000 $\mathrm{m}$ for TTIDE station 8) in the right panel of (a). Strain is normalized by its 200-300-m value in the right panel of (b), showing departures from uniformity only as the seafloor is approached at sites of energetic tidal generation or reflection such as HOME Nearfield and TTIDE.

If true, these harmonics account for approximately $2 / 3$ of the strain variance in the sea, given the Munk (1981) spectral model of the wave field. ${ }^{2}$ Such forced harmonics do not obey linear internal wave scaling relations. Yet there is no need to posit an additional class of motions to explain this discrepancy. The fact that the simulated waveform is strongly reminiscent of the data in Figs. 7 and 8 reinforces the interpretation of these motions as distorted waves.

Transitioning from the study of a single nonlinear wave to an ocean thermocline governed by Poisson statistics is the present challenge. An odd aspect of this simulation might be of relevance. In a frame moving at the wave phase speed, the velocity field is limited between $0<c_{X}-U \leq u \leq c_{x}+U$. Thus, the largest and smallest strains that can be experienced are

$$
\begin{aligned}
& \gamma_{\max }=c_{x} /\left(c_{x}-U\right), \\
& \gamma_{\min }=c_{x} /\left(c_{x}+U\right)>1 / 2 .
\end{aligned}
$$

While $\gamma_{\max }$ can get very large as the wave orbital speed approaches the phase speed, $\gamma_{\min }$ is always greater than 0.5 , as seen in the probability density functions of Fig. 9b. This odd limit might not be present when multiple wave packets are modeled in more realistic environments. But it is inherent here. ${ }^{3}$ The fact that the simulated PDF in Fig. 9b is so narrow offers the promise that the strain field associated with a superposition of independent nonlinear waves, with probability density function given by the convolution of the PDFs of

\footnotetext{
${ }^{2}$ Neglecting contributions from the vortical field.

${ }^{3}$ As an alternative perspective, $\gamma=0.5$ or $\gamma^{2}=0.25$ represents a kinematic limit on the convective instability of a single plane wave. The similarity between this and the Miles-Howard threshold for shear instability is notable.
}

the individual waves, might yield the exponential distribution that is at the heart of the Poisson model of the thermocline. In this present (during COVID-19 shelter-in-place) analytical exploration of wave distortion, the signature of a sum of independent sinusoids cannot be modeled; such a sum is not an exact solution to the fully nonlinear equations of motion. Furthermore, this simulation does not address the small-scale form of the oceanic shear spectrum, which shares many of the characteristics of the strain spectrum. More realistic examples of wave-induced thermocline distortion can be simulated numerically.

\section{Variation with depth/buoyancy frequency}

Statistically, most metrics of wavefield variability for the seven Pacific datasets are in accord with general experience. If vertical displacement variance $\left\langle\hat{\eta}^{2}\right\rangle$ in $200-\mathrm{m}$ depth bands is plotted as a function of squared buoyancy frequency $\overline{N^{2}}$ (Fig. 10a, left), there is an increase in variance with decreasing $\overline{N^{2}}$. In Fig. 10a (right), the variance in each band is normalized by its respective value in the 200-400-m (1500-1700 $\mathrm{m}$ for the deep TTIDE observations) band, removing much of the siteto-site variability in the mean buoyancy frequency $\overline{N^{2}}$. The tendency for displacement variance to scale as $\left(\overline{N^{2}}\right)^{-1 / 2}$ shows through clearly, being consistent with linear internal wave theory in the WKB approximation. Distinct departures from WKB scaling are seen in the HOME Nearfield and TTIDE data, where intense near-seafloor tidal generation and reflection occur.

The corresponding variance of $2-\mathrm{m}$ strain $(\overline{\Delta z}=2 \mathrm{~m})$ is presented as a function of $\overline{N^{2}}$ in Fig. 10b (left). Strain variance at each site is remarkably constant as $\overline{N^{2}}$ varies by a factor of 50 . When strain variance at each site is normalized by its value in the 200-400-m depth bin, right, the pan-Pacific data collapse to a single line. There is a near-seafloor strain enhancement in 
the energetic TTIDE site and also at the lower-energy AESOP site. However, there is none at the high-energy Nearfield site, where the deepest observations are $300 \mathrm{~m}$ above the crest of Kaena Ridge. Nearfield displacement variance, in contrast, shows a pronounced increase with depth.

The observed constancy of strain variance with depth is not in accord with the behavior of linear waves in the WKB approximation. It is closely tied to the observation that the highwavenumber cutoff in the vertical strain (Fig. 6) and shear spectra at approximately $k_{c}^{-1}=10 \mathrm{~m}$ remains constant with depth at any given open ocean site. WKB scaling mandates that $k_{c}$ vary linearly with the buoyancy frequency. This violation of WKB, the constancy of $k_{c}$ with depth, was incorporated by Munk in the 1981 version of the Garret-Munk spectral model (Munk 1981). This brought the model into accord with the growing body of observations at that time, as well as those presented here.

\section{Discussion}

The principal finding of this work is that the vertical straining of the Pacific thermocline is observed to obey Poisson statistics over a range of vertical scales from $\kappa_{0}^{-1}(\sim 0.5-2 \mathrm{~m})$ to $Z_{\text {corr_sL }}$ ( $\sim 100-200 \mathrm{~m})$. Here $Z_{\text {corr_sL }}$ is the vertical correlation scale of isopycnal vertical displacement, defined as the first zero of the displacement correlation function

$$
R_{\eta}\left(Z_{\text {corr_sL }}\right)=\left\langle\hat{\eta}(z) \hat{\eta}\left(z+Z_{\text {corr_sL }}\right)\right\rangle \equiv 0 .
$$

Both isopycnal displacement and strain vary in depth/ $\overline{N^{2}}(z)$ in a manner consistent with the Munk (1981) version of the GM model at the open ocean sites (Fig. 10). The constancy of 2-m strain variance with depth implies that the Poisson constant $\kappa_{0}$ is itself independent of depth (Table 3). It follows that all higher moments of strain over all separation scales in the open ocean Poisson subrange are $\operatorname{depth} / \overline{N^{2}}(z)$ independent.

When vertical wavenumber spectra of strain from across the Pacific are rescaled by $\kappa_{0}$ and plotted against dimensionless vertical wavenumber $k / \kappa_{0}$, variability of a factor of 20 is reduced to a factor of 2 . In demonstrating this rescaling, no reference to the background state of the ocean, e.g., $\overline{N^{2}}(z)$, or even to linear internal wave dynamics is invoked. The rescaling procedure appears effective in both open ocean situations, where $\kappa_{0}$ is depth independent, and at sites where the near-seafloor wave field is greatly enhanced by generation or reflection.

A random-walk statistical model of the thermocline is found to replicate thermocline structure with considerable realism. The ability to generate synthetic vertical profiles of scalars is of great use in simulating issues as diverse as plankton dynamics and population distributions, acoustic propagation, etc. In a vertically homogeneous ocean, the model thermocline is composed of a stack of Poisson elements, each of mean vertical extent $\kappa_{0}^{-1}$. The fact that one can superpose any mean scalar profile on this uniform stack, including, for example, a highly variable density profile which should strongly refract linear waves (Fig. 10a), and see no consequences in how these scalars are strained (Fig. 10b) attests to the ability of the model to replicate key nonlinear aspects of the wave field.
An unsettling aspect of the model is that Poisson element boundaries are inherently unknowable. An experiment cannot be devised to identify the boundaries between regions of uniform strain variation. This is because the irregularity of the thermocline is not a property of the water but rather of the wave field. Realizations of the steps are due to individual wave groups; step statistics are a property of the wavefield climate, as established by local forcing, dissipation and refraction. In a homogeneous wave field, an empirical ability to identify fixed Poisson boundaries cannot be expected.

As vertical scales approach the Poisson scale $\kappa_{0}^{-1}$, the observed PDFs of strain (Fig. 3) begin to deviate from the Poisson-gamma model. Structure functions of separation (Part II, Fig. 1) also deviate from predicted Poisson form (Table 3). While instrument resolution plays some role in this departure, the more fundamental issue is the imposition of human metrics of deformation on the Poisson thermocline. For example, two closely spaced isopycnal surfaces will experience quite different separation statistics, depending on whether the pair resides within the same Poisson element or not. If this uncertainty is taken as fundamental, simple calculations lead to a set of Poisson microscale relationships that parallel the classical results of Table 3 . However, the microscale results extend to arbitrary small vertical separations, whereas the classical results apply only for $\kappa_{0} \overline{\Delta z} \geq 1$. Example calculations of Poisson microscale behavior are presented in appendix $\mathrm{C}$ for semi-Lagrangian second- and third-order structure functions of isopycnal separation. The Eulerian vertical wavenumber spectrum of scalar gradients, as presented in Fig. 5, is also derived. Surprisingly, some microscale effects extend to arbitrarily large vertical scale, although the magnitude of the departure from classical Poisson behavior diminishes.

A key microscale prediction is that isopycnal separation variance grows as $\overline{\Delta z}^{2}$ at separations $\overline{\Delta z} \ll \kappa_{0}^{-1}$. The data support this prediction (Part II, Fig. 1a). This is significant because any model of the thermocline based on summing statistically independent thickness layers will exhibit a variance that grows linearly with separation, regardless of the probability law governing the layers. The conjecture is that at scales below $\kappa_{0}^{-1}$ the physics of straining transitions from an additive process to a multiplicative one, as originally explored by Batchelor (1959) for isotropic turbulence.

Oceanic strain is a mix of vortical and internal wave motions. In these Pacific Ocean measurements, small-vertical-scale waves are the dominant signal (see appendix B; Pinkel 2014). If one considers an internal wave with vertical wavelength $50 \mathrm{~m}$ propagating through a thermocline with $\overline{N^{2}}=1 \mathrm{cph}^{2}$, the horizontal phase speed is about $50 \mathrm{~m} \mathrm{~h}^{-1} \sim 0.014 \mathrm{~m} \mathrm{~s}^{-1}$. Such a wave is strongly affected by variation in the background shear. When vertical propagation is inhibited, wave amplitude grows. Such arrested waves appear as distinct strain features embedded in the background current with signatures that slope across the density field. A simple simulation of a large-amplitude plane internal wave, Fig. 9, demonstrates a strain signature that is qualitatively similar to the ocean observations of Figs. 7 and 8. Importantly, the strain field associated with a sinusoidal velocity field can be highly skewed, more skewed than the exponential distribution that is the limiting form of the gamma 
probability family. It is thus plausible that a thermocline strained by multiple wave packets at varying stages of interaction with the background shear could be associated with a strain field that is well modeled as a Poisson random walk.

More accurate modeling of the small-scale wave field can be used to address this issue. Analytically, Chunchuzov (1996, 2002) has extended the pioneering approach of Allen and Joseph (1989) to model both weak resonant interactions and the stronger effects of self-distortion and Doppler shifting. While his approach is challenging to implement, selected case studies could complement further numerical exploration of the cascade, perhaps replicating the Poisson-like nature of the ocean thermocline. Including forced nonresonant interactions along with resonant interactions is key to the realistic modeling of the oceanic wave field.

Figures 7 and 8 show the role played by near-inertial shear along with near-inertial and tidal strain (Sun and Kunze 1999) in modulating the propagation of short internal waves. When shear inhibits the vertical propagation of a wave packet, local wave amplitude grows and thermocline steps can develop. One-quarter inertial period later, the tailwind of the background flow subsides and the packet resumes propagation, returning to a more sinusoidal waveform. The formation of these adiabatic steps in conjunction with a causal background shear must play a key role in orchestrating the presence of overturning and resultant ocean mixing. Ideally, an awareness of the Poisson nature of the thermocline will catalyze advances in our understanding of wave interactions and the energy cascade.

Acknowledgments. The author thanks Eric Slater, Lloyd Green, Mike Goldin, Tony Aja, Chris Neely, Mai Bui, Tyler Hughen, San Nguyen, Jonathan Ladner, and Sara Goheen for their participation in the development of the instrument systems described here and for the operation of these systems at sea. The seagoing leadership of Captains DeWitt Efird and Tom Golfinos (R/P FLIP) and Tom Desjardins and Dave Murline (R/V Roger Revelle) is greatly appreciated. Mike Gregg, Matthew Alford, Jennifer MacKinnon, Andrew Lucas, and Gregory Wagner provided valuable comments on drafts of this work. Continued support from the National Science Foundation and the Office of Naval Research is gratefully acknowledged.

\section{APPENDIX A}

\section{The Joint Probability Density of Vertical Strain and Displacement}

Knowledge of the joint PDF of strain and displacement is of value in many applications. The challenge is to develop a model in which the difference in the depths of any two density surfaces is described by the gamma PDF while the sum (average) of the depths is Gaussian.

Let $\hat{\eta}_{1}, \hat{\eta}_{2}$ represent the vertical displacements of density surfaces separated by $\overline{\Delta z}$ in the mean. Here $z$ is positive upward, and $\eta_{1}$ is above $\eta_{2}$. Both have zero mean, as defined here. Thus, the density surfaces will cross if $\hat{\eta}_{2}-\overline{\Delta z}>\hat{\eta}_{1}$.

The PDFs of $\hat{\eta}_{1}$ and $\hat{\eta}_{2}$ are Gaussian, with a correlation that decreases with increasing mean separation. One can define orthogonal variables:

$$
\begin{gathered}
\Delta z=\hat{\eta}_{1}-\hat{\eta}_{2}+\overline{\Delta z}, \\
\bar{\eta}=\left(\hat{\eta}_{1}+\hat{\eta}_{2}\right) / 2 .
\end{gathered}
$$

With $\hat{\eta}$ Gaussian, the correlation function $\hat{R}_{\eta}(\overline{\Delta z})=R_{\eta}(\overline{\Delta z}) / R_{\eta}(0)$ is the appropriate second-order descriptor of the relatedness of isopycnal pairs. The correlation function both sets the rate of increase of isopycnal separation variance and the rate of decrease of the mean isopycnal displacement variance with mean separation:

$$
\begin{aligned}
\left\langle\Delta z^{2}\right\rangle-\overline{\Delta z}^{2} & =2\left\langle\hat{\eta}^{2}\right\rangle\left[1-\hat{R}_{\eta_{-} P}(\overline{\Delta z})\right] \\
& =\overline{\Delta z} / \kappa_{0} \quad \text { (Poisson model) },
\end{aligned}
$$

in the Poisson subrange, or, anticipating Part II,

$$
\begin{aligned}
\left\langle\Delta z^{2}\right\rangle-\overline{\Delta z}^{2} & =2\left\langle\hat{\eta}^{2}\right\rangle\left[1-\hat{R}_{\eta_{-G M}}(\overline{\Delta z})\right] \\
& =2\left\langle\hat{\eta}^{2}\right\rangle\left[1-\exp \left(-\frac{\overline{\Delta z}}{2 \kappa_{0}\left\langle\hat{\eta}^{2}\right\rangle}\right)\right] \quad \text { (GM model) } .
\end{aligned}
$$

Also,

$$
\left\langle\bar{\eta}^{2}\right\rangle=\left\langle\hat{\eta}^{2}\right\rangle\left[1+\hat{R}_{\eta}(\overline{\Delta z})\right] / 2 .
$$

It follows from (A3a) and (A3b) that

$$
\hat{R}_{\eta_{-} P}(\overline{\Delta z})=1-\overline{\Delta z} /\left(2 \kappa_{0}\left\langle\hat{\eta}^{2}\right\rangle\right), \quad \overline{\Delta z} \leq 2 \kappa_{0}\left\langle\hat{\eta}^{2}\right\rangle \quad \text { (Poisson), }
$$

$$
\hat{R}_{\eta_{\mathrm{GM} P}}(\overline{\Delta z})=\exp \left(-\frac{\overline{\Delta z}}{2 \kappa_{0}\left\langle\hat{\eta}^{2}\right\rangle}\right) \quad(\mathrm{GM})
$$

and

$$
\left\langle\bar{\eta}^{2}\right\rangle \equiv \sigma_{\bar{\eta}}^{2}=\left\langle\hat{\eta}^{2}\right\rangle\left[1+\hat{R}_{\eta_{-} P}(\overline{\Delta z})\right] / 2=\left\langle\hat{\eta}^{2}\right\rangle-\overline{\Delta z} /\left(4 \kappa_{0}\right) \quad \text { (Poisson) },
$$

$$
\left\langle\bar{\eta}^{2}\right\rangle \equiv \sigma_{\bar{\eta}}^{2}=\left\langle\hat{\eta}^{2}\right\rangle\left\{1+\exp \left[-\left(\overline{\Delta z}^{2} / 2 \kappa_{0}\left\langle\hat{\eta}^{2}\right\rangle\right)\right]\right\} / 2 \quad(\mathrm{GM}) .
$$

The variance $\left\langle\bar{\eta}^{2}\right\rangle$ is exactly equal to $\left\langle\hat{\eta}^{2}\right\rangle$ when density surfaces are closely spaced and $\hat{R}_{\eta}(\overline{\Delta z})=1$. The variance drops to $1 / 2\left\langle\hat{\eta}^{2}\right\rangle$ for uncorrelated density surfaces.

The idealized Poisson correlation function goes negative at

$$
Z_{\text {corr_sL }} \equiv 2 \kappa_{0}\left\langle\hat{\eta}^{2}\right\rangle
$$

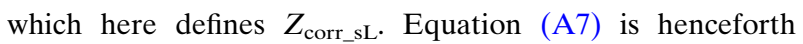
termed the Poisson wavefield relation (see Part II).

By expanding the modified GM_P correlation function

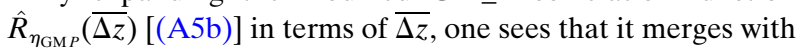
the Poisson correlation at small separations. It also avoids the ever-increasing negativity of the Poisson correlation function at large separations, providing a seamless link between Poisson scales and the larger-scale motions in the thermocline. 
Consider now a joint PDF for independent strain and displacement of the separable form

$$
P\left(\Delta z, \bar{\eta} \mid \overline{\Delta z}, \sigma_{\bar{\eta}}^{2}\right)=G_{\Delta z}(\Delta z \mid \overline{\Delta z}) P_{\bar{\eta}}\left(\bar{\eta} \mid \sigma_{\bar{\eta}}^{2}\right) .
$$

We require $P_{\bar{\eta}}\left(\bar{\eta} \mid \sigma_{\bar{\eta}}^{2}\right)$, the probability density function of $\bar{\eta}$, to be Gaussian,

$$
P_{\bar{\eta}}\left(\bar{\eta} \mid \sigma_{\bar{\eta}}^{2}\right)=\left(2 \pi \sigma_{\bar{\eta}}^{2}\right)^{-1 / 2} \exp \left\{-\left[\bar{\eta}^{2} /\left(2 \sigma_{\bar{\eta}}^{2}\right)\right]\right\}
$$

with variance given by (A6a) and (A6b).

For tracking the separation of density surfaces, we use the gamma probability density, as supported by the extensive body of observations reported here.

$$
G_{\Delta z}(\Delta z \mid \overline{\Delta z})=\kappa_{0}\left(\kappa_{0} \Delta z\right)^{\kappa_{0} \overline{\Delta z}-1} e^{-\kappa_{0} \Delta z} / \Gamma\left(\kappa_{0} \overline{\Delta z}\right) .
$$

The resulting joint PDF is

$$
\begin{aligned}
P\left(\Delta z, \bar{\eta} \mid \overline{\Delta z}, \sigma_{\bar{\eta}}^{2}\right)= & G_{\Delta z}(\Delta z \mid \overline{\Delta z}) P_{\bar{\eta}}\left(\bar{\eta} \mid \sigma_{\bar{\eta}}^{2}\right) \\
P\left(\Delta z, \bar{\eta} \mid \overline{\Delta z}, \sigma_{\bar{\eta}}^{2}\right)= & \frac{1}{\sqrt{2 \pi \sigma_{\bar{\eta}}^{2}} \Gamma\left(\kappa_{0} \overline{\Delta z}\right)}\left\{\kappa_{0}\left(\kappa_{0} \Delta z\right)^{\kappa_{0} \overline{\Delta z}-1}\right. \\
& \left.\times e^{-\kappa_{0} \Delta z} \exp \left[-\bar{\eta}^{2} /\left(2 \sigma_{\bar{\eta}}^{2}\right)\right]\right\}
\end{aligned}
$$

Using the GM_P expression for $\sigma_{\bar{\eta}}^{2}$ enables the PDF to be valid for all scales $\kappa_{0} \overline{\Delta z}>1$.

To evaluate this PDF, one must pick any two parameters from $\kappa_{0}, Z_{\text {corr_sL }}$, and $\left\langle\hat{\eta}^{2}\right\rangle$ and evaluate the third using the Poisson wavefield relation (A7). The joint PDF can then be calculated at any $\overline{\Delta z}$.

Integrating (A11) over $\bar{\eta}$, one recovers the PDF of separation, which is of gamma form and depends only on $\kappa_{0}$. Similarly, integrating over $\Delta z$ yields the PDF of mean displacement, $\bar{\eta}$, which is Gaussian. This PDF depends on any two of the

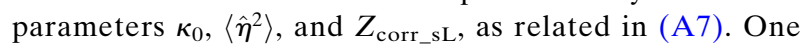
can rotate the coordinates of this 2D PDF and integrate over $\eta_{1}$ to verify that the PDF of $\eta_{2}$ is Gaussian, with variance $\left\langle\hat{\eta}^{2}\right\rangle$.

Unlike a jointly normal PDF, here the probability that the density surfaces can cross is zero. This is a critical feature of a "reversible finestructure" model. Also, unlike a jointly normal PDF, the statistics of separation (strain) are highly skewed in a manner that matches data from across the Pacific.

\section{APPENDIX B}

\section{Internal Waves, Quasigeostrophic Motions, and the Poisson Thermocline}

Early attempts to apply theoretical internal wave consistency relations to model the vertical coherences of horizontal and vertical velocity were unsuccessful. It was posited that an additional field of velocity and density "fine structure" must be present in the thermocline to produce the observed coherence patterns. The lead-off paper in the first issue of the Journal of Physical Oceanography, Phillips (1971), addresses this issue. The Garrett and Munk (1972) internal wave spectral model was necessarily preceded by a Garrett and Munk (1971) fine structure spectral model. This model enabled consistent determination of both wave and fine structure model parameters from observations. Not until a decade later was a dynamical legitimacy provided to fine structure. In a seminal work, Muller (1984), identified fine structure as the manifestation of quasi geostrophic signals at internal wave spatial scales. He termed these motions the vortical mode, emphasizing their role in advectively transporting potential vorticity and distinguishing them from the wave modes that propagate energy vertically.

Subsequently (Pinkel 2008) it was appreciated that the vertical advection of both vortical and small-scale wave signals by larger internal scale waves contribute to the discrepancy with consistency relations. This random "vertical Doppler shifting", can be modeled by convolving an "initial spectrum" with a function whose width is determined by the variance of the advecting velocities. This contrasts with the early approach of adding a modeled fine structure spectrum to the initial spectrum. In the convolution formalism, the need to postulate an additional energetic fine structure field to explain observations is greatly reduced.

When working in an isopycnal following frame, only the effects of horizontal Doppler shifting are present. The vortical signal can be identified in wavenumber-frequency spectra of strain as a spectral ridge centered at zero frequency and extending across vertical wavenumbers $-0.1<$ $k<0.1 \mathrm{cpm}$ (Pinkel 2014). If a fixed aspect ratio is assumed for the vortical field at all scales, lateral advection will lead to a Doppler shifted frequency-bandwidth of the ridge that grows with increasing wavenumber magnitude. This indeed is seen. A surprisingly "flat" aspect ratio, of order $0.1 f / N$, is inferred for vortical motions at a variety of Pacific Ocean sites.

The internal wave signal can be separated from the vortical ridge using simple linear filters. Depth-time examples of the total strain field and wave-only strain are presented in Figs. $7 \mathrm{~b}$ and $7 \mathrm{~d}$. Wavenumber and frequency spectral estimates for the separated wave and vortical strain fields are presented in Pinkel (2014). Typically, the vortical signal comprises $10 \%-$ $30 \%$ of the overall strain variance. In open ocean observations, the contribution is most prominent at smaller vertical scales and shallow depths.

From the perspective of the Poisson model, the vortical signal should formally be considered an aspect of the rest-state density profile, a preexisting feature that is passively strained by the internal wave field. However, given the effects of lateral advection, multiple realizations of the vortical field are seen in the course of a multiday field effort. Advection converts the vortical field from background to signal.

Examination of the statistics of the individual wave and vortical fields is possible, e.g., Fig. 7d, although beyond the scope of the present work. Recall that if the two fields are statistically independent, the probability density function of the combined field represents the convolution of the PDFs of the individual fields. If one of these signals has extremely small variance relative to the other, its PDF will have a delta-like 
form. The convolution will return an accurate depiction of the PDF of the high variance field.

If the variance of the vortical and wave fields in the thermocline is comparable, the form of their individual PDFs must somehow be such that their convolution yields the observed gamma probability distribution (Fig. 3). This robust result cannot be dependent on the relative magnitudes of the independent fields, which clearly vary with depth and location. However, if each field individually is described by a gamma probability distribution, their convolution is also a gamma probability density. The variance is the sum of the individual field variances. To the extent that the vortical strain at one depth does not enable prediction of vortical strain at adjacent depths beyond some distance $\kappa_{V}^{-1}$, the vortical straining of the thermocline might well be Poisson.

With two Poisson fields contributing to the signal the observed decrease in strain variance with separation,

$$
\left\langle\hat{\gamma}^{2}\right\rangle=1 /\left(\kappa_{W} \overline{\Delta z}\right)+1 /\left(\kappa_{V} \overline{\Delta z}\right)
$$

would still follow the $\overline{\Delta z}^{-1}$ form that is observed PA92. The presence of the vortical field simply leads to estimates of $\kappa_{0}$ that reflect the combined contributions of wave and vortical motions,

$$
1 / \kappa_{0}=\left\langle\hat{\gamma}^{2}\right\rangle \overline{\Delta z}=1 /\left\langle\hat{\gamma}^{2}\right\rangle(\overline{\Delta z})=1 / \kappa_{W}+1 / \kappa_{V} .
$$

A similar result is obtained from examination of the mean cube strain. While the presence of vortical signals does not necessarily diminish the applicability of the Poisson model, the converse is that one cannot isolate separate wave and vortical contributions based on vertical profile statistics alone. Fortunately, the strain wavenumber frequency spectrum effectively distinguishes the fields when observations are made in isopycnal coordinates. Analyses of these separated fields (e.g., Fig. 7d) await a future work.

\section{APPENDIX C}

\section{The Poisson Microscale: Sampling a Highly Skewed Thermocline}

The non-Gaussian nature of the thermocline challenges both experiment design and interpretation. In PA92, it was noted that, if a set of density surfaces separated in the mean by $\kappa_{0}^{-1}$ in an sL frame is tracked in an Eulerian frame, different pairs of surfaces will bound any given reference depth from realization to realization. The time average separation of the pairs of surfaces bounding the fixed depth is $2 \kappa_{0}^{-1}$, twice the sL result. This odd discrepancy stems from the fact that any given depth spends more time in low-gradient "layers" than in highgradient "sheets." If one chooses to describe thermocline variability in terms of a set of $n$ isopycnals separated by some $\overline{\Delta z} \sim \kappa_{0}^{-1}$ in an sL frame, the sum of the Eulerian mean distances between the surfaces would be greater than $(n-1) \overline{\Delta z}$. The variances in separation are greater, also, but the difference is not as pronounced. Thus the normalized Eulerian strain variance, the separation variance divide by the Eulerian mean, squared, is half its sL counterpart. In PA92, Eulerian strain estimates were obtained by selecting fixed depths and tracking the separation between the nearest isopycnals that bound the depths from realization to realization. The corresponding PDFs of Eulerian strain matched their analytical forms with precision similar to the sL observations (PA92, their Figs. 4 and 5).

In standard oceanographic practice, it is more common to quantify the Eulerian frame thermocline deformation in terms of the departure of the local density gradient from the large (vertical) scale mean. If a time average of gradient fluctuations $1 / \Delta z(t)$ is formed in an Eulerian frame, the resulting Eulerian mean gradient is just the inverse of a time average of $\Delta z(t)$ formed in the sL frame, $\overline{\Delta z}^{-1}$. Thus there is no gross discrepancy between the sL approach and Eulerian metrics commonly used in oceanography.

In applying the Poisson model, a complicating factor is that one observes the evolution of the thermocline in terms of human-imposed metrics, such as the time fluctuations of density at a set of fixed depths or the depth variability of a set of specified densities. By design, these metrics fall at random relative to any conceptual Poisson structure of the thermocline and have statistics that differ slightly from the idealized Poisson model. The differences become significant as scales approach $\kappa_{0}^{-1}$, where ocean vertical strain is correlated and in phase. The departure of observed probability density functions of separation in Fig. 3 and in second- and third-order structure functions (Figs. 1, A1, and A2 in Part II) from the Poisson model motivates a closer examination of this issue.

Here, "sampled Poisson" or "microscale" derivations are presented for the variance, mean cube, and autocovariance of isopycnal vertical separation. These are based on tracking density surfaces of arbitrary separation $\overline{\Delta z}$, transcending the limit $\kappa_{0} \overline{\Delta z} \geq 1$ of the Poisson-gamma model.

\section{a. The semi-Lagrangian Poisson microscale}

Within an individual Poisson element, strain has unity variance, and for any two density surfaces within the element,

$$
\begin{aligned}
\left\langle\Delta \hat{\eta}^{2}\right\rangle & \equiv\left\langle(\Delta \eta-\overline{\Delta z})^{2}\right\rangle=\overline{\Delta z}^{2}, \\
\left\langle\Delta \eta^{2}\right\rangle & =2 \overline{\Delta z}^{2},
\end{aligned}
$$

and

$$
\begin{aligned}
\left\langle(\Delta \eta-\overline{\Delta z})^{3}\right\rangle & =\left\langle\Delta \hat{\eta}^{3}\right\rangle=2 \overline{\Delta z}^{3}, \\
\left\langle\Delta \eta^{3}\right\rangle & =6 \overline{\Delta z}^{3} .
\end{aligned}
$$

Given a known sL background profile, two reference densities with mean separation $\overline{\Delta z}$ have a probability $1-\kappa_{0} \overline{\Delta z}$ of falling within the same Poisson element and experiencing the above separation variability. If the densities span the boundary between elements the separation variance is

$$
\left\langle\Delta \hat{\eta}^{2}\right\rangle=\delta_{1}^{2}+\delta_{2}^{2},
$$

where $\delta_{1}$ is the distance between the first density surface and the Poisson boundary (in the mean profile) and $\delta_{2}=\overline{\Delta z}-\delta_{1}$ is 
the corresponding distance of the second surface from the boundary. From realization to realization, the corresponding independent random variables $\widehat{\delta_{1}}$ and $\widehat{\delta_{2}}$ are uniformly distributed, with domains $\left\{0, \kappa_{0}^{-1}-\overline{\Delta z}\right\},\{0, \overline{\Delta z}\}$, respectively. Averaging over these domains, one finds

$$
\begin{aligned}
\left\langle\Delta \hat{\eta}^{2}\right\rangle & =\overline{\Delta z^{2}}-1 / 3 \kappa_{0} \overline{\Delta z}^{3}, \quad \kappa_{0} \overline{\Delta z} \leq 1, \\
& =\overline{\Delta z} / \kappa_{0}-1 / 3 \kappa_{0}^{-2}, \quad \kappa_{0} \overline{\Delta z} \geq 1, \\
\left\langle\Delta \hat{\eta}^{3}\right\rangle & =2 \overline{\Delta z}-\kappa_{0} \overline{\Delta z}^{4}, \quad \kappa_{0} \overline{\Delta z} \leq 1, \\
& =2 \overline{\Delta z} / \kappa_{0}^{2}-\kappa_{0}^{-3}, \quad \kappa_{0} \overline{\Delta z} \geq 1 .
\end{aligned}
$$

The initial term in these expressions matches the corresponding property of the gamma PDF, valid for scales $\overline{\Delta z}>\kappa_{0}^{-1}$. The tracer-derived moments are always less than their gamma model counterparts, and this offset extends to arbitrarily large vertical scales. However, when skewness, the ratio of third and second moments is formed, the influence of these offsets vanishes at large scale. The microscale separation variance and skewness track the second- and third-order structure functions (Fig. 1 in Part II), far better than the expressions associated with the classical gamma PDF.

Isopycnal separation variance $\left\langle\Delta \hat{\eta}^{2}\right\rangle$ is related to the associated Poisson covariance of vertical displacement $R_{\eta P \mu}(\overline{\Delta z})$ by

$$
\left\langle\Delta \hat{\eta}^{2}\right\rangle(\overline{\Delta z})=2\left\langle\hat{\eta}^{2}\right\rangle-2 R_{\eta}(\overline{\Delta z}) .
$$

Combining (C6) and (C8) yields

$$
\begin{array}{ll}
R_{\eta P \mu}(\overline{\Delta z})=\left\langle\hat{\eta}^{2}\right\rangle-\overline{\Delta z}^{2} / 2+\kappa_{0}\left|\overline{\Delta z}^{3}\right| / 6, & \kappa_{0} \overline{\Delta z} \leq 1, \\
R_{\eta P \mu}(\overline{\Delta z})=\left\langle\hat{\eta}^{2}\right\rangle-\overline{\Delta z} /\left(2 \kappa_{0}\right)+1 /\left(6 \kappa_{0}^{2}\right), & \kappa_{0} \overline{\Delta z}>1 .
\end{array}
$$

Anticipating the discussion in Part II, the Poisson microscale displacement covariance $R_{\eta P \mu}$ can be approximated by a covariance function of exponential form:

$$
\begin{aligned}
R_{\eta \mathrm{GM} \mu}(\overline{\Delta z}) & =\left\langle\hat{\eta}_{*}^{2}\right\rangle \exp \left[-\left(|\overline{\Delta z}|^{2}-1 / 3 \kappa_{0}|\overline{\Delta z}|^{3}\right) /\left(2\left\langle\hat{\eta}_{*}^{2}\right\rangle\right)\right], \\
\kappa_{0} \overline{\Delta z} & \leq 1 \\
R_{\eta \mathrm{GM} \mu}(\overline{\Delta z}) & =\left\langle\hat{\eta}_{*}^{2}\right\rangle \exp \left[-\left(|\overline{\Delta z}|-1 / 3 \kappa_{0}^{-1}\right) /\left(2 \kappa_{0}\left\langle\hat{\eta}_{*}^{2}\right\rangle\right)\right], \\
\kappa_{0} \overline{\Delta z} & >1 .
\end{aligned}
$$

Here, strictly non-Gaussian considerations stemming from the exponential nature of the Poisson gamma PDF at scales $\kappa_{0} \overline{\Delta z} \sim 1$ have led to the detail in the displacement covariance at small $\overline{\Delta z}$. In Part II the identical expressions are again obtained from model covariance functions of strain and displacement, a strictly second-order derivation. When Fourier transformed, this microscale detail sets the high-wavenumber spectral cutoff, $k=k_{c}$.

These Poisson-derived expressions exactly replicate the GM_P covariance functions (24a) and (24b) in Part II, with the subtle distinction that $\left\langle\hat{\eta}^{2}\right\rangle$ in Eq. (24) in Part II represents the displacement variance that the GM spectrum would have had if a high-wavenumber cutoff had not been imposed. The variance $\left\langle\hat{\eta}_{*}^{2}\right\rangle$ is the true wavefield variance. The two definitions differ by the factor $\exp \left[1 /\left(6 \kappa_{0}^{2}\left\langle\hat{\eta}_{*}^{2}\right\rangle\right)\right]$.

\section{b. The Eulerian microscale of vertical gradients}

It is common in oceanography to be presented with a single realization of a scalar profile and to infer strain from the departure of this realization from an assumed "undistorted", background profile. Strain is defined as the ratio $\mu=(\partial \theta / \partial z) /(\overline{\partial \theta / \partial z})$, where $\theta$ represents a scalar variable and the overbar denotes smoothing in the vertical (e.g., Polzin et al. 1995). In terms of the Poisson model of the thermocline,

$$
\begin{aligned}
\mu\left(z_{0}, t\right) & =\left[\Delta \theta / \Delta z\left(z_{0}, t\right)\right] /\left\{\Delta \theta\left\langle 1 /\left[\Delta z\left(z_{0}, t\right)\right]\right\rangle_{E}\right\} \\
& =\left[1 / \Delta z\left(z_{0}, t\right)\right] /\left\langle 1 / \Delta z\left(z_{0}, t\right)\right\rangle_{E} \\
& =\overline{\Delta z} / \Delta z\left(z_{0}, t\right) .
\end{aligned}
$$

Here, $\Delta z\left(z_{0}, t\right)$ represents the separation of that pair of tracers that is spanning depth $z_{0}$ at time $t$ and the brackets \langle\rangle$_{E}$ represent an average across realizations of those pairs of density surfaces that span $z_{0}$.

The Eulerian covariance and vertical wavenumber spectrum of $\mu$ are derived by considering gradient variability across some fixed vertical separation $H=Z_{b}-Z_{a}$ (PA92, section 2). Over some fraction of the realizations, the entire interval $H$ will fall within a single Poisson element and the vertical gradients at $Z_{b}$ and $Z_{a}$ will be identical. In the Poisson model, the distances between the bounding Poisson elements and the upper and lower boundaries of $H$,

$$
\delta_{a}=Z_{a}-z_{i} \text { and } \delta_{b}=z_{i+1}-Z_{b}
$$

are independent random variables, each governed by the exponential probability distribution. The probability density of the total separation $\left(\delta_{a}+\delta_{b}+H\right)$ of the Poisson element that bound $H$ is given by

$$
P_{\text {tot }} E(\Delta z \mid H)=\kappa_{0}\left[\kappa_{0}(\Delta z-H)\right] e^{-\left[\kappa_{0}(\Delta z-H)\right]}, \quad H<\Delta z<\infty .
$$

To quantify $\mu$ it is necessary to first determine

$$
\begin{aligned}
\nu_{1} & \equiv\left\langle\frac{1}{\Delta z}\right\rangle=\kappa_{0}^{2} \int_{H}^{\infty} \Delta z^{-1}(\Delta z-H) e^{-\kappa_{0}(\Delta z-H)} d \Delta z \\
& =\kappa_{0}\left[1-\kappa_{0} H e^{\kappa_{0} H} E_{1}\left(\kappa_{0} H\right)\right],
\end{aligned}
$$

and

$$
\begin{aligned}
\nu_{2} & \equiv\left\langle\frac{1}{\Delta z^{2}}\right\rangle=\kappa_{0}^{2} \int_{H}^{\infty} \Delta z^{-2}(\Delta z-H) e^{-\kappa_{0}(\Delta z-H)} d \Delta z \\
& =\kappa_{0}^{2} e^{\kappa_{0} H}\left(1+\kappa_{0} H\right) E_{1}\left(\kappa_{0} H\right)-\kappa_{0}^{2} .
\end{aligned}
$$

Here, $E_{1}\left(\kappa_{0} H\right)$ is the exponential integral function. The variance of $\mu$ is

$$
\left\langle\hat{\mu}(H)^{2}\right\rangle=\left\langle\mu^{2}\right\rangle-\langle\mu\rangle^{2}=v_{2} / v_{1}^{2}-1
$$

Gradient values at the upper and lower boundaries of $H$ will fluctuate with this variance. They fluctuate identically only 
when $H$ does not span a Poisson boundary. Otherwise the fluctuations are independent. The probability that no Poisson boundaries (points) fall within $H$ is just $P_{0}=e^{-\kappa_{0} H}$. Thus, the Eulerian covariance of vertical gradients is

$$
\begin{aligned}
& R_{\mu E}(H)=\left\langle\hat{\mu}(H)^{2}\right\rangle e^{-\kappa_{0}|H|}=\left(v_{2} / v_{1}^{2}-1\right) e^{-\kappa_{0}|H|}, \\
& -\infty<H<\infty, \quad H \neq 0 .
\end{aligned}
$$

The associated vertical wavenumber spectrum of vertical gradient fluctuations, $S_{\mu E}(k)$, is obtained by Fourier transformation of $R_{\mu E}(H)$. Analytically, this process is complicated by the fact that $\left\langle\hat{\mu}(0)^{2}\right\rangle$ and $R_{\mu E}(0)$ are unbounded, corresponding to infinite gradient variance. This is an issue shared with the GM 1981 spectrum and with other spectral models that are matched to the observational literature. It stems from the fact that the gradient spectrum decays at high wavenumber at a rate $k^{-1}$ or slower. The singularity at $R_{\mu E}(0)$ is fortunately tractable, because, in general, only integrals of the gradient covariance are of interest. If we require that the vertical displacement spectrum is related to the gradient spectrum by $S_{\eta P}(k)=$ $(2 \pi k)^{-2} S_{\mu}(k)$, asserting that the Eulerian gradient is a proper measure of $\partial \eta / \partial z$, then $R_{\mu E}=-R_{\eta P}^{\prime \prime}$. Integrating this expression once, the integral of $R_{\mu E}$ across the singularity at $H=0$ is equal to the negative of the change in slope of $R_{\eta P}$. From (C9b), the slope of $R_{\eta P}$ is just $-1 /\left(2 \kappa_{0}\right)$ for $H>\kappa_{0}^{-1}$ and the change in slope across $H=0$ is just $-\kappa_{0}^{-1}$. Contributions to this slope change come from both the singularity at $H=0$ and the detailed structure of $R_{\mu E}(H)$ (C17) at small scales. In simulating this numerically, one can set the value of $R_{\mu E}(0)$ to that of an "integrated delta function" of magnitude

$$
R_{\mu E}(0)=\left(1.5 \pi \kappa_{0} d H\right)^{-1},
$$

where $d H$ is the resolution increment in the simulation. The resulting gradient covariance function can be Fourier transformed to generate an Eulerian strain spectrum that is properly related to the displacement spectrum through multiplication by $(2 \pi k)^{-2}$ (Fig. 5a, red curves). All other aspects of the Poisson model are preserved as well. To account for finite sensor resolution, $R_{\mu E}$ can be convolved by the squared sensor spatial response function $\Psi^{2}(H)$ prior to Fourier transformation.

\section{REFERENCES}

Allen, K. R., and R. I. Joseph, 1989: A canonical statistical theory of oceanic internal waves. J. Fluid Mech., 204, 185-228, https:// doi.org/10.1017/S0022112089001722.

Batchelor, G. K., 1959: Small-scale variation of convected quantities like temperature in turbulent fluid Part 1. General discussion and the case of small conductivity. J. Fluid Mech., 5, 113-133, https://doi.org/10.1017/S002211205900009X.

Bretherton, F. P., 1966: The propagation of groups of internal gravity waves in a shear flow. Quart. J. Roy. Meteor. Soc., 92, 466-480, https://doi.org/10.1002/qj.49709239403.

Broutman, D., 1984: The focusing of short internal waves by an inertial wave. Geophys. Astrophys. Fluid Dyn., 30, 199-225, https://doi.org/10.1080/03091928408222850.
Cairns, J. L., and G. O. Williams, 1976: Internal wave observations from a midwater float, 2. J. Geophys. Res., 81, 1943-1950, https://doi.org/10.1029/JC081i012p01943.

Chunchuzov, I. P., 1996: The spectrum of high-frequency internal waves in the atmospheric wave-guide. J. Atmos. Sci., 53, 17981814, https://doi.org/10.1175/1520-0469(1996)053<1798:TSOHFI> 2.0.CO;2.

_ 2002: On the high-wavenumber form of the Eulerian internal wave spectrum in the atmosphere. J. Atmos. Sci., 59, 17531774, https://doi.org/10.1175/1520-0469(2002)059<1753:OTHWFO > 2.0.CO;2.

Desaubies, Y. J., and M. C. Gregg, 1981: Reversible and irreversible finestructure. J. Phys. Oceanogr., 11, 541-556, https:// doi.org/10.1175/1520-0485(1981)011<0541:RAIF > 2.0.CO;2.

Garrett, C., and W. Munk, 1971: Internal wave spectra in the presence of fine structure. J. Phys. Oceanogr., 1, 196-202, https://doi.org/10.1175/1520-0485(1971)001<0196:IWSITP> 2.0.CO;2.

—_, and ——, 1972: Space-time scales of internal waves. Geophys. Fluid Dyn., 3, 225-264, https://doi.org/10.1080/ 03091927208236082.

—, and —, 1975: Space-time scales of internal waves: A progress report. J. Geophys. Res., 80, 291-297, https://doi.org/ 10.1029/JC080i003p00291.

Gill, A., 1982: Atmosphere-Ocean Dynamics. Academic Press, $662 \mathrm{pp}$.

Gregg, M. C., 1989: Scaling turbulent dissipation in the thermocline. J. Geophys. Res., 94, 9686-9698, https://doi.org/10.1029/ JC094iC07p09686.

Hayes, S. P., T. M. Joyce, and R. C. Millard, 1975: Measurements of vertical fine structure in the Sargasso Sea. J. Geophys. Res., 80, 314-319, https://doi.org/10.1029/JC080i003p00314.

Henyey, F. S., J. Wright, and S. M. Flatté, 1986: Energy and action flow through the internal wave field: An eikonal approach. J. Geophys. Res., 91, 8487, https://doi.org/ 10.1029/JC091iC07p08487.

Kunze, E., 2017: Internal-wave-driven mixing: Global geography and budgets. J. Phys. Oceanogr., 47, 1325-1345, https:// doi.org/10.1175/JPO-D-16-0141.1.

Muller, P., 1984: Small-scale vortical motions. Internal Gravity Waves and Small Scale Turbulence: Proc. 'Aha Huliko'a Hawaiian Winter Workshop, Honolulu, HI, University of Hawai'i at Mānoa, 249-261.

Munk, W. H., 1981: Internal waves and small-scale processes. Evolution of Physical Oceanography, B. A. Warren and C. Wunsch, Eds., MIT Press, 264-291.

Orlanski, I., and K. Bryan, 1969: The formation of thermocline step structure by large-amplitude internal gravity waves. J. Geophys. Res., 74, 6975-6983, https://doi.org/ 10.1029/JC074i028p06975.

Papoulis, A., 1984: Probability, Random Variables and Stochastic Processes. McGraw-Hill, 576 pp.

Phillips, O. M., 1966: The Dynamics of the Upper Ocean. Cambridge University Press, 261 pp.

_- 1971: On spectra measured in an undulating layered medium. J. Phys. Oceanogr., 1, 1-6, https://doi.org/10.1175/ 1520-0485(1971)001<0001:OSMIAU > 2.0.CO;2.

Pinkel, R., 1975: Upper ocean internal wave observations from FLIP. J. Geophys. Res., 80, 3892-3910, https://doi.org/10.1029/ JC080i027p03892.

- 2008: Advection, phase distortion, and the frequency spectrum of finescale fields in the sea. J. Phys. Oceanogr., 38, 291313, https://doi.org/10.1175/2007JPO3559.1. 
, 2014: Vortical and internal wave shear and strain. J. Phys. Oceanogr., 44, 2070-2092, https://doi.org/10.1175/JPO-D-13-090.1. , and S. Anderson, 1992: Toward a statistical description of finescale strain in the thermocline. J. Phys. Oceanogr., 22, 773-795, https://doi.org/10.1175/1520-0485(1992)022<0773: TASDOF $>2.0 . \mathrm{CO} ; 2$.

— , and — 1997: Shear, strain, and Richardson number variations in the thermocline. Part I: Statistical description. J. Phys. Oceanogr., 27, 264-281, https://doi.org/10.1175/15200485(1997)027<0264:SSARNV>2.0.CO;2.

_ 2020: The Poisson link between internal wave and dissipation scales in the thermocline. Part II: Internal waves, overturns, and the energy cascade. J. Phys. Oceanogr., 50, 3425-3438, https://doi.org/10.1175/JPO-D-19-0287.1.

Polzin, K. L., J. M. Toole, and R. W. Schmitt, 1995: Finescale parameterizations of turbulent dissipation. J. Phys. Oceanogr.,
25, 306-328, https://doi.org/10.1175/1520-0485(1995)025<0306: FPOTD $>2.0 . C O ; 2$.

Stommel, H., and K. N. Fedorov, 1967: Small scale structure in temperature and salinity near Timor and Mindanao. Tellus, 19, 306-325, https://doi.org/10.3402/tellusa.v19i2.9792.

Sun, H., and E. Kunze, 1999: Internal wave-wave interactions: Part I. The role of internal wave vertical divergence. J. Phys. Oceanogr., 29, 2886-2904, https://doi.org/10.1175/1520-0485(1999) 029<2886:IWWIPI >2.0.CO;2.

Whalen, C. B., L. D. Talley, and J. A. MacKinnon, 2012: Spatial and temporal variability of global ocean mixing inferred from Argo profiles. Geophys. Res. Lett., 39, L18612, https://doi.org/ 10.1029/2012GL053196.

Woods, J. D., 1968: Wave-induced shear instability in the summer thermocline. J. Fluid Mech., 32, 791-800, https://doi.org/10.1017/ S0022112068001035. 